\title{
Particle Acceleration in Relativistic Outflows
}

\author{
Andrei Bykov • Neil Gehrels • Henric Krawczynski • \\ Martin Lemoine · Guy Pelletier · Martin Pohl
}

Received: 26 March 2012 / Accepted: 10 May 2012 / Published online: 3 June 2012

(C) Springer Science+Business Media B.V. 2012

\begin{abstract}
In this review we confront the current theoretical understanding of particle acceleration at relativistic outflows with recent observational results on various source classes thought to involve such outflows, e.g. gamma-ray bursts, active galactic nuclei, and pulsar wind nebulae. We highlight the possible contributions of these sources to ultra-high-energy cosmic rays.
\end{abstract}

\footnotetext{
A. Bykov

Ioffe Institute of Physics and Technology, 194021 St. Petersburg, Russia e-mail: byk@astro.ioffe.ru
}

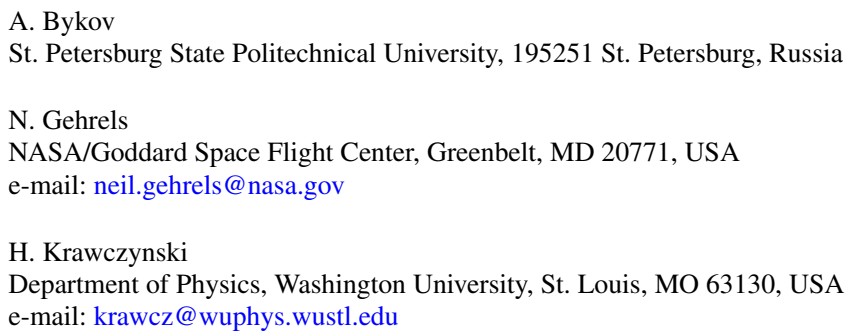

M. Lemoine

Institut d'Astrophysique de Paris, CNRS-UPMC, 98bis boulevard Arago, 75014 Paris, France e-mail: lemoine@iap.fr

G. Pelletier Institut de Planétologie et d'Astrophysique de Grenoble, BP 53, 38041 Grenoble Cédex 9, France e-mail: Guy.Pelletier@obs.ujf-grenoble.fr

\section{Pohl (ه)}

Institut für Physik und Astronomie, Universität Potsdam, 14476 Potsdam, Germany e-mail: marpohl@uni-potsdam.de

M. Pohl

DESY, 15738 Zeuthen, Germany

e-mail: martin.pohl@desy.de 
Keywords Cosmic rays $\cdot$ Particle acceleration $\cdot$ Shocks

\section{Introduction}

High-energy astrophysical phenomena stem from the generation of powerful flows emanating from supernova explosions, gamma-ray bursts (GRB), from ejections in the environment of black holes or neutron stars that lead to the formation of very strong shocks, at which particle acceleration takes place. The new developments in these issues, especially for relativistic shocks, are based on the interdependence between the shock structure, the generation of supra-thermal particles and the generation of turbulence. It is thought, and numerical simulations support that view, that the penetration of supra-thermal particles in the shock precursor generates magnetic turbulence which in turn provides the scattering process needed for particle acceleration through the Fermi process. This successful development, first elaborated for supernova remnants (SNR), inspired similar investigations for the termination shock of GRBs. However, in ultra-relativistic shocks, difficulties arise with the transverse magnetic field that places a limitation to particle penetration upstream and that drags particles in the downstream flow and makes shock recrossing difficult. It turns out that only sufficiently fast micro-turbulence can make the Fermi process operative, as demonstrated by recent numerical simulations. Following a review of the main observational results on GRBs, active galactic nuclei (AGN), and pulsar wind nebulae (PWN), these points are briefly discussed and astrophysical consequences are drawn. We describe the role relativistic shocks inside relativistic flows, e.g. the internal shocks of the prompt-emission stage of GRBs, may play in the generation of ultra-high-energy cosmic rays (UHECR). Noting that the energy required for supplying sub-GZK UHECR is huge compared with the available energy budget, we also discuss other sources, such as AGN and young pulsars, that may contribute to the flux at ultra-high energies, all the more so if the composition is enriched in heavy nuclei, as suggested by recent experimental results.

\section{Gamma Ray Bursts}

The first GRB was observed by one of the Vela satellites monitoring for the Nuclear Test Ban Treaty in 1967, but the (unexpected) astronomical results were not declassified and published for another six years (Klebesadel et al. 1973). For many years the nature of GRBs was unknown, since the distance scale was completely unknown. Beginning about 20 years ago, the cosmological spatial distribution of GRBs was strongly hinted at due to the very isotropic distribution on the sky of GRBs localized by CGRO. The wealth of detailed information garnered in the last seven years by Swift (Gehrels et al. 2004) has taken the study of GRBs to the next level, and indeed the current situation is in some sense more confusing than our naive pre-Swift picture (e.g. Gehrels et al. 2009).

In this subsection we (i) review the basic properties of the two main types of GRBs, long and short, (ii) look at long GRBs in more detail, (iii) review the brief history of short GRBs and the difficulties entailed in their study, (iv) provide an overview of the acceleration processes for GRB jets, and (v) conclude with a recent results on high energy emission observed by Fermi. 
Fig. 1 Representative broad-band $\nu F_{v}$ spectra (Gehrels et al. 2009) of a lGRB (910503) (Kaneko et al. 2007) and a sGRB (980425) (Kaneko et al. 2008) along with the Crab pulsar nebula (Kuiper et al. 2001) and Cyg X-1 (McConnell et al. 2002)

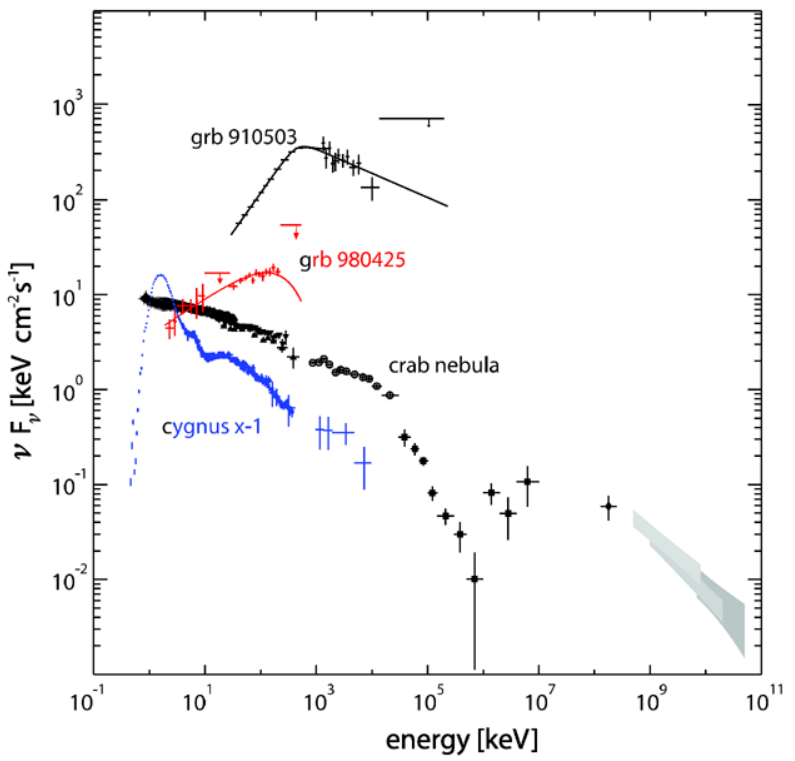

\subsection{GRB Properties}

GRBs come in two kinds, long and short, where the dividing line between the two is $\sim 2 \mathrm{~s}$ (Kouveliotou et al. 1993). Long GRBs (GRBs) are thought to be due to the collapse of a massive star, while short GRBs (sGRBs) are inferred to be neutron star - neutron star (NSNS) mergers. A further division can be made spectrally according to their hardness ratio (i.e., ratio of high to low energies). The redshift range is from about 0.2 to 2 for sGRBs, with a mean of about 0.4. For lGRBs the range is between about 0.009 and 8.2, with a mean of about 2.3. The typical energy release is $\sim 10^{49}-10^{50} \mathrm{erg}$ for sGRBs and $\sim 10^{50}-10^{51} \mathrm{erg}$ for lGRBs. These ranges are based on observed isotropic-equivalent energies of $\sim 10^{51} \mathrm{erg}$ for sGRBs and $\sim 10^{53} \mathrm{erg}$ for lGRBs, and estimates for jet beaming for each class, $\theta_{\mathrm{j}} \sim 5^{\circ}$ for lGRBs and $\theta_{\mathrm{j}} \sim 15^{\circ}$ for sGRBs (Burrows et al. 2006; Grupe et al. 2006). Beaming angles for sGRBs are still highly uncertain. The corresponding beaming factors $f_{b}=1-\cos \theta_{\mathrm{j}} \simeq$ $\theta_{\mathrm{j}}^{2} / 2$ are roughly $1 / 300$ for lGRBs and $1 / 30$ for sGRBs. The sGRBs have weaker X-ray afterglows, a mean value of $\sim 7 \times 10^{-10} \mathrm{erg} \mathrm{cm}^{-2} \mathrm{~s}^{-1}$ versus $\sim 3 \times 10^{-9} \mathrm{erg} \mathrm{cm}^{-2} \mathrm{~s}^{-1}$ for lGRBs. Figure 1 shows spectra for several representative GRBs, and two other high-energy sources, the Crab nebula and Cyg X-1.

\subsection{LGRBs}

The BeppoSAX mission made the critical discovery of X-ray afterglows of long bursts (Costa et al. 1997). With the accompanying discoveries by ground-based telescopes of optical (van Paradijs et al. 1997) and radio (Frail et al. 1997) afterglows, lGRBs were found to emanate from star forming regions in host galaxies at typical distance of $z \simeq 1-2$. BeppoSAX and the following HETE-2 mission also found evidence of associations of GRBs with Type Ic $\mathrm{SNe}$. This supported the growing evidence that lGRBs are caused by "collapsars" where the central core of a massive star collapses to a black hole (MacFadyen and Woosley 1999).

LGRBs are incredibly bright. A typical galaxy at a redshift of only $z=3$ is fainter than $m \simeq 27$. Multiwavelength observations of the current record holder, GRB 090432 (at $z \simeq 8$ ), 
Table 1 High $z$ GRBs

\begin{tabular}{llllll}
\hline$z$ & $t_{\mathrm{LB}}(\mathrm{Gyr})$ & GRB & \multicolumn{3}{l}{ Optical brightness } \\
\hline 8.3 & 13.0 & 090423 & $K=20$ & $@$ & $20 \mathrm{~min}$ \\
6.7 & 12.8 & 080813 & $K=19$ & $@$ & $10 \mathrm{~min}$ \\
6.29 & 12.8 & 050904 & $J=18$ & $@$ & $3 \mathrm{~h}$ \\
5.6 & 12.6 & 060927 & $I=16$ & $@$ & $2 \mathrm{~min}$ \\
5.3 & 12.6 & 050814 & $K=18$ & $@$ & $23 \mathrm{~h}$ \\
5.11 & 12.5 & 060522 & $R=21$ & $@$ & $1.5 \mathrm{~h}$ \\
\hline
\end{tabular}

are providing information about the universe at a time when it was only about $4 \%$ of its current age, and shed light on the process of reionization in the early universe (Tanvir et al. 2009; Salvaterra et al. 2009). The highest redshift GRBs are seen to have high luminosity, resulting in fluxes well above the detection threshold. Such bursts are also strong at other wavelengths. Table 1 presents optical data for the highest redshift GRBs observed to date, where the look-back time $t_{\mathrm{LB}}(\mathrm{Gyr})$ is given in column 2 .

\subsection{SGRBs}

At Swift's launch, the greatest mystery of GRB astronomy was the nature of short-duration, hard-spectrum bursts. Although more than 50 lGRBs had afterglow detections, no afterglow had been found for any sGRB. Swift provided the first sGRB X-ray afterglow localization with GRB 050509B and HETE-2 enabled the first optical afterglow detection with GRB 050709. These two bursts, plus Swift observations of GRB 050824, led to a breakthrough in our understanding (Gehrels et al. 2005; Bloom et al. 2006; Fox et al. 2005; Villasenor et al. 2005; Hjorth et al. 2005; Barthelmy et al. 2005; Berger et al. 2005) of sGRBs. BAT has now detected 60 sGRBs, most of which have XRT detections, and about one third of which have host identifications or redshifts (an additional two have been detected by HETE-2, one by INTEGRAL, and two by Fermi/LAT). We now have $\sim 50$ sGRB localizations.

In stark contrast to long bursts, the evidence to date on short bursts is that they can originate from regions with low star formation rate. GRB 050509B and 050724 were from elliptical galaxies with low current star formation rates while GRB 050709 was from a region of a star forming galaxy with no nebulosity or evidence of recent star formation activity in that location. Recent HST observations of locations of sGRBs in their hosts reveal that sGRBs trace the light distribution of their hosts while lGRBs are concentrated in the brightest regions (Fong et al. 2010). SGRBs are also different from lGRBs in that accompanying supernovae are not detected for nearby events (Bloom et al. 2006; Fox et al. 2005; Hjorth et al. 2005). Taken together, these results support the interpretation that short bursts are associated with an old stellar population, and may arise from mergers of compact binaries [i.e., double neutron star or neutron star-black hole (NS-BH) binaries].

\subsection{GeV Emission}

Fermi was launched into low-Earth orbit in June 2008 and has two primary high-energy detectors: the Large Area Telescope (LAT) which operates between $20 \mathrm{MeV}$ and $2300 \mathrm{GeV}$, and the Gamma-Ray Burst Monitor (GBM) which operates between $8 \mathrm{keV}$ and $40 \mathrm{MeV}$. So far the LAT has detected 24 GRBs; two were sGRBs, and nine showed extended emission. The emission from GRB 090902B included a $34 \mathrm{GeV}$ photon. One of the most luminous to date has been GRB 080916C (Abdo et al. 2009a) at a redshift of 4.35. It had extended 
emission (18 min) and exhibited a lag in LAT energies with respect to GBM. GRB 090510 is unique in being the only short burst with a known redshift (0.903) showing $\mathrm{GeV}$ emission. The lack of detectable time delay between specific peaks in the light curves of GRB 090510 at different energies leads to strong constraints on Lorentz invariance (Abdo et al. 2009b). Recent theoretical work (Kumar and Barniol Duran 2010) on the Fermi/LAT detected GRBs suggests that these may represent unusually powerful explosions with Lorentz factors $\gtrsim 10^{3}$ in which the entire progenitor is obliterated. The simplest model, namely an external shock with synchrotron emission, can be used to take the early values (at $\sim 10-10^{2}$ s) of the observed high-energy emission and successfully predict the much later values of the optical and X-ray afterglow (at $\sim 10^{5}-10^{6} \mathrm{~s}$ ).

\subsection{GRB Summary}

Recent progress in GRB research has been strongly motivated by observational discoveries. To date, Swift has detected about 600 GRBs, Fermi/LAT 24. High redshift GRBs are illuminating the properties of the high- $z$ universe and probing into the era of re-ionization. Swift finds sGRBs in different environments than lGRBs; also sGRBs are not accompanied by supernovae. The accumulating evidence provides support for the NS-NS merger model. Many GRBs have delayed onset of GeV emission, and more have extended high energy emission. Interesting constraints on the Lorentz factors associated with outflow, and Lorentz invariance violation, also come from the synergism between $\mathrm{GeV}$ and lower energy observations. It is not currently know for certain whether GRB jets are made primarily of baryons or Poynting flux, but momentum for the latter idea appears to be gaining strength.

\subsection{Jet Launching Processes}

There are currently two primary lines of thought regarding the creation and propagation of jets in GRBs.

The baryonic jet model, whose roots can be traced back to the elegant analytical solutions of a relativistic blast wave by Blandford and McKee (1976), posits that a jet containing about a Jupiter's mass worth of gas, $\sim 0.001 M_{\odot}$, is somehow launched near the BH created by the collapsar with a Lorentz factor $\Gamma \simeq 10-20$ (Zhang et al. 2003). The jet propagates through the dense stellar envelope of the progenitor star where it is focussed and compressionally heated. After breaking free of the stellar surface, the thermal energy of the compressed jet is translated into bulk kinetic motion, further accelerating the jet to a Lorentz factor of several hundred. Subsequent deceleration by the circumstellar medium of the jet, which is then idealized as being the fragment of a relativistic shell so that the Blandford \& McKee formalism can be brought to bear (Sari et al. 1998), can then be used to infer jet beaming angles from putative achromatic "breaks" in the GRB decay light curves (Frail et al. 2001).

The Poynting flux jet model, which has been gaining momentum in recent years, traces its roots back to Blandford and Znajek (1977) who considered the electromagnetic (EM) extraction of energy from within the ergosphere of a Kerr BH. In the last few years workers have developed sophisticated numerical codes that calculate the 3D evolution of gas and EM fields from the inner edge of accretion disks onto spinning BHs, taking into account both general relativity and magnetohydrodynamics (McKinney 2005; McKinney and Narayan 2007a, 2007b). The accretion disk inner edge provides a natural collimating surface. These workers find a baryonic zone-of-exclusion within the jet, which effectively suppresses any baryonic component. For numerical stability the numerical codes need to have some mass within each grid point, therefore a small trace amount of matter is constantly added within 
grid points where the EM fields try to exclude it. These are called "floor" models. Previous studies that attempted to place constraints on the jet Lorentz factors, which were based on the baryonic jet assumption, have been called into question. The ramifications on the jet dynamics of having a predominantly Poynting flux jet have not been developed yet in any detail.

\section{Nonthermal Particle Acceleration in GRBs: Challenges and Perspectives}

Physical mechanisms of prompt emission in GRBs are still to be established. There are fundamental questions of the powerful relativistic outflow composition and matter vs. magnetic field dominance to be addressed with both observations and advanced models. The relativistic outflows may be different for two types of GRB progenitors under consideration. Long and soft GRBs are most likely connected to supernovae, while the short and hard GRBs are possibly related to compact binary mergers.

Magnetized outflows from GRB engines of different nature have been studied for some decades (see, e.g. Usov 1992; Thompson 1994; Mészáros and Rees 1997; Lyutikov and Blandford 2004; Zhang and Yan 2011). The outflows converting the rotation power of a compact collapsar into a broad band of radiation are likely models of GRBs and AGNs. The principal question here is our understanding of the microscopic mechanisms of the conversion of magnetic field energy into non-thermal particles and the observed emission-with or without shock formation. Anisotropic striped wind with alternating magnetic polarity is considered a favorable configuration to convert the magnetic energy into the observed radiation of pulsar wind nebulae (e.g. Kirk et al. 2009; Sironi and Spitkovsky 2011). An alternating magnetic field configuration may occur in relativistic jets of GRBs. Magnetic field reconnection demonstrated to produce electric fields that accelerate particles in the Earth magnetosphere, solar flares, and some laboratory plasma configurations (see e.g. Yamada et al. 2010) was also proposed as a plausible GRB model by (e.g. Spruit et al. 2001; Lyutikov and Blandford 2004; Giannios and Spruit 2005; Zhang and Yan 2011; McKinney and Uzdensky 2012).

The most elaborated model of the origin of the GRB prompt emission is, by now, the relativistic dissipative fireball model. There are a number of alternative particle acceleration and radiation processes within the relativistic dissipative fireball paradigm (see e.g. Rees and Meszaros 1994; Piran 2004; Mészáros 2006; Mimica and Aloy 2012). Synchrotron and inverse Compton radiation in the optically thin regions of the relativistic fireball can be associated with the non-thermal electron/positron accelerated either in the internal dissipation processes due to shocks or to the flow magnetic field reconnections. Another potential component is the photospheric emission (thermal or non-thermal) that is rather rarely identified in the observed GRB emission. The prompt emission light curves and spectra are generally in agreement with the internal shock models. However, some potential problems of the scenario are its efficiency and the lack of a bright photospheric component observed in a few GRBs. We shall discuss now some general features of the internal dissipation models with emphasis on particle acceleration processes.

\subsection{Energetic Efficiency of Internal Dissipation Models: Shell Collisions in Jets}

An important issue of the internal dissipation scenario where the energy carried out by multiple colliding shells of different magnetization is the efficiency of the conversion of the outflow power into the observed radiation. The variability of the central engine (of a timescale 
$t_{\mathrm{var}}$ ) driving the relativistic outflows of the mean Lorentz factor $\Gamma$, either matter or Poynting dominated, can be modelled as a collision of energy-containing shells. The model is considered to explain the main features of the GRB prompt emission (see e.g. Piran 2004; Mészáros 2006), as a vital alternative to the photospheric models of GRBs. The dissipation region typically exists at the radii about $r_{\text {diss }} \sim c t_{\text {var }} \Gamma^{2}$. In the case of matter dominated jets (of low magnetization) the inner dissipation occurs in the internal shocks while in the electromagnetically (Poynting flux) dominated jets the magnetic field reconnection effects are most likely crucial though shocks may also occur. The microphysics of the dissipation in relativistic shocks as well as modeling of the magnetic field reconnections are under intense studies (Yamada et al. 2010; Bykov and Treumann 2011; Sironi and Spitkovsky 2011; McKinney and Uzdensky 2012). Realistic models of a jet that would simulate the global RMHD dynamics and simultaneously resolve the dissipative microphysical plasma processes at much smaller spatial scales are not feasible at the moment. However, simple multiple shell models of the internal dissipation that just parameterize the magnetic field reconnection effects are still rather useful and it is instructive to discuss some of these models. A similar approach can be applied to other relativistic outflows, like those of the AGN jets (see for a discussion Sect. 4) and of the pulsar wind nebulae.

To illustrate the effect of the outflow magnetization $\sigma=B^{2} / 4 \pi \Gamma \rho c^{2}$ on the energy conversion into the observed radiation it is instructive to use a simple two shell model (see e.g. Panaitescu et al. 1999; Kumar 1999; Daigne and Mochkovitch 1998; Zhang and Yan 2011). The shell collision may result in dissipation of the magnetic energy due to reconnection and turbulence cascade. Consider two shells of masses, Lorentz factors and magnetization parameters $\left[\left(\left(m_{1}, \Gamma_{1}, \sigma_{1}\right)\right.\right.$ and $\left.\left.\left(m_{2}, \Gamma_{2}\right), \sigma_{2}\right)\right]$, respectively, colliding inelastically with the formation of a merged shell of $\left(m_{\mathrm{f}}, \Gamma_{\mathrm{f}}, \sigma_{\mathrm{f}}\right)$. The internal energy $\delta^{\prime}$ released in the rest frame of the merged shell (of the Lorentz factor $\Gamma_{\mathrm{f}}$ ) is assumed to be in the form of either thermal or non-thermal accelerated particles and radiation with nearly isotropic distribution in the rest frame. Then, in the observer frame the released energy is $\Delta E=\Gamma_{\mathrm{f}} \delta^{\prime}$. This leads to conversion of some amount of magnetic energy into internal energy of the fluid, and then to radiation. Let us envisage a picture where the two shells merge with a lower magnetization parameter $\sigma_{\mathrm{f}}$ by the end of such an inelastic collision. Energy conservation and momentum conservation can be presented as

$$
\begin{aligned}
& \Gamma_{1} \Psi_{1} m_{1}+\Gamma_{2} \Psi_{2} m_{2}=\Gamma_{\mathrm{f}} \Psi_{\mathrm{f}}\left(m_{1}+m_{2}+\delta^{\prime}\right)-\frac{\gamma-1}{\gamma \Gamma_{\mathrm{f}}} \delta^{\prime}, \\
& \Psi_{\mathrm{i}}\left(\Gamma_{\mathrm{i}}\right)=1+\frac{2 \Gamma_{\mathrm{i}}^{2}-1}{2 \Gamma_{\mathrm{i}}^{2}} \sigma_{\mathrm{i}}
\end{aligned}
$$

and

$$
\Gamma_{1} \beta_{1} m_{1}\left(1+\sigma_{1}\right)+\Gamma_{2} \beta_{2} m_{2}\left(1+\sigma_{2}\right)=\Gamma_{\mathrm{f}} \beta_{\mathrm{f}}\left(m_{1}+m_{2}+\delta^{\prime}\right)\left(1+\sigma_{\mathrm{f}}\right) .
$$

Since the merged shell can not be considered as a cold one anymore

$$
\delta^{\prime}=\frac{\left(P^{\prime}+\rho_{\mathrm{f}}^{\prime}\right) V^{\prime}}{c^{2}},
$$

where $\rho_{\mathrm{f}}^{\prime}$ is the proper kinetic energy density (i.e. the internal energy density with the rest energy density subtracted), $V^{\prime}$ is the shell volume, and $P^{\prime}$ is the pressure in the rest frame of the merged shell. Note that to calculate the energy released in the rest frame of the merged shell $\delta^{\prime}$ one should keep all of the terms of the order of $\Gamma_{\mathrm{i}}^{-2}$. The expression for $\Psi_{\mathrm{i}}\left(\Gamma_{\mathrm{i}}\right)$ is 


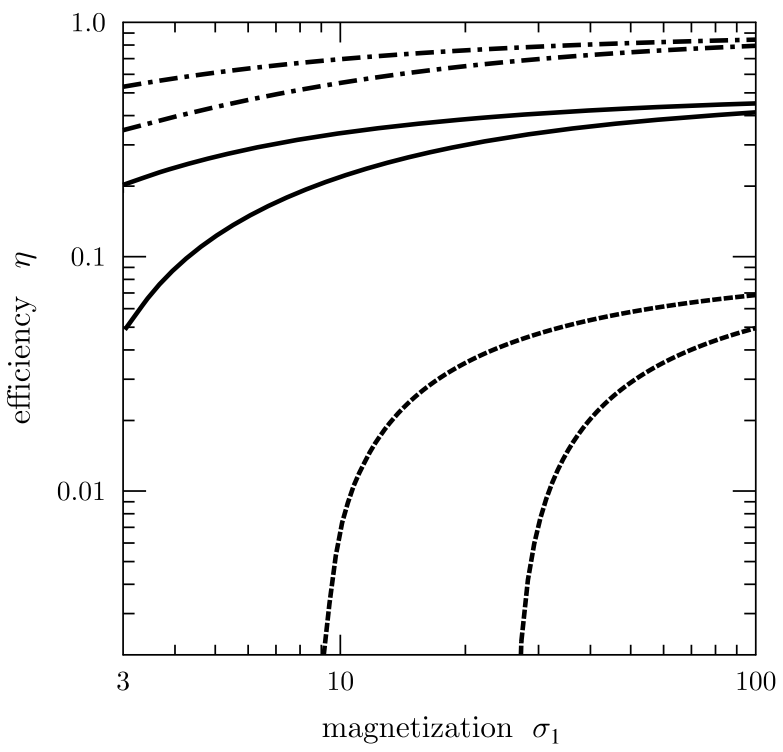

Fig. 2 Simulated energy dissipation efficiency $\eta$ defined by Eq. (5) as a function of the shell magnetization parameter $\sigma$ for the inelastic collision of two initially cold magnetized shells of equal masses, but of different Lorentz factors $\left(\Gamma_{1}=500\right.$ and $\left.\Gamma_{2}=1000\right)$. The key parameter is the final magnetization of the hot merged shells $\sigma_{\mathrm{f}}$ was chosen to be 0.1 (dot-dashed curves), 1.0 (solid lines) and 10.0 (dashed lines). The two curves of the same line style in the Figure differ by the assumed initial magnetization parameters. The top curve for each line style corresponds to the case of equal initial magnetization $\sigma_{1}=\sigma_{2}$, while the lower curves of each type correspond to the fixed $\sigma_{2}=0.1$. The adiabatic index $\gamma=4 / 3$ was fixed for the matter in the hot merged shell

exact for the case of the transverse magnetic field in the rest frame of a shell, and it accounts for the energy of the induced electric fields in the observer frame.

In general, there are a few distinct components that contribute to the pressure and the proper energy density (thermal and nonthermal baryons, leptons, and photons). If for simplicity we describe these as a single fluid with adiabatic index $\gamma$, then

$$
P^{\prime}+\rho_{\mathrm{f}}^{\prime}=\frac{\gamma P^{\prime}}{\gamma-1}
$$

and one can resolve the energy-momentum conservation equations, assuming the simple equation of state Eq. (4) to calculate the Lorentz factor of the merged shell $\Gamma_{\mathrm{f}}$ and the energy dissipation efficiency $\eta$ of the inelastic collision of the two cold magnetized shells

$$
\eta=\frac{\Gamma_{\mathrm{f}} \delta^{\prime}}{\Gamma_{1} \Psi_{1} m_{1}+\Gamma_{2} \Psi_{2} m_{2}} .
$$

In Fig. 2 we show the simulated energy dissipation efficiency $\eta$ as a function of the shell magnetization parameter $\sigma$ for the inelastic collision of two initially cold magnetized shells of equal masses, but of different Lorentz factors. We consider the GRB jet as a generic case and therefore choose $\Gamma_{1}=500$ and $\Gamma_{2}=1000$. The energy dissipation efficiency $\eta$, the mean enthalpy of the hot matter in the merged shell, that characterizes the mean Lorentz factor of the randomized particles in the rest frame of the hot merged shell (shown in Fig. 3), and the Lorentz factor $\Gamma_{\mathrm{f}}$ of the hot merged shell in the observer frame, shown in Fig. 4, are derived 
Fig. 3 Simulated dimensionless mean enthalpy that is characterizing the mean Lorentz factor of the randomized particles in the rest frame of a hot merged shell. The curves are simulated for the same parameter sets as it is indicated in Fig. 2
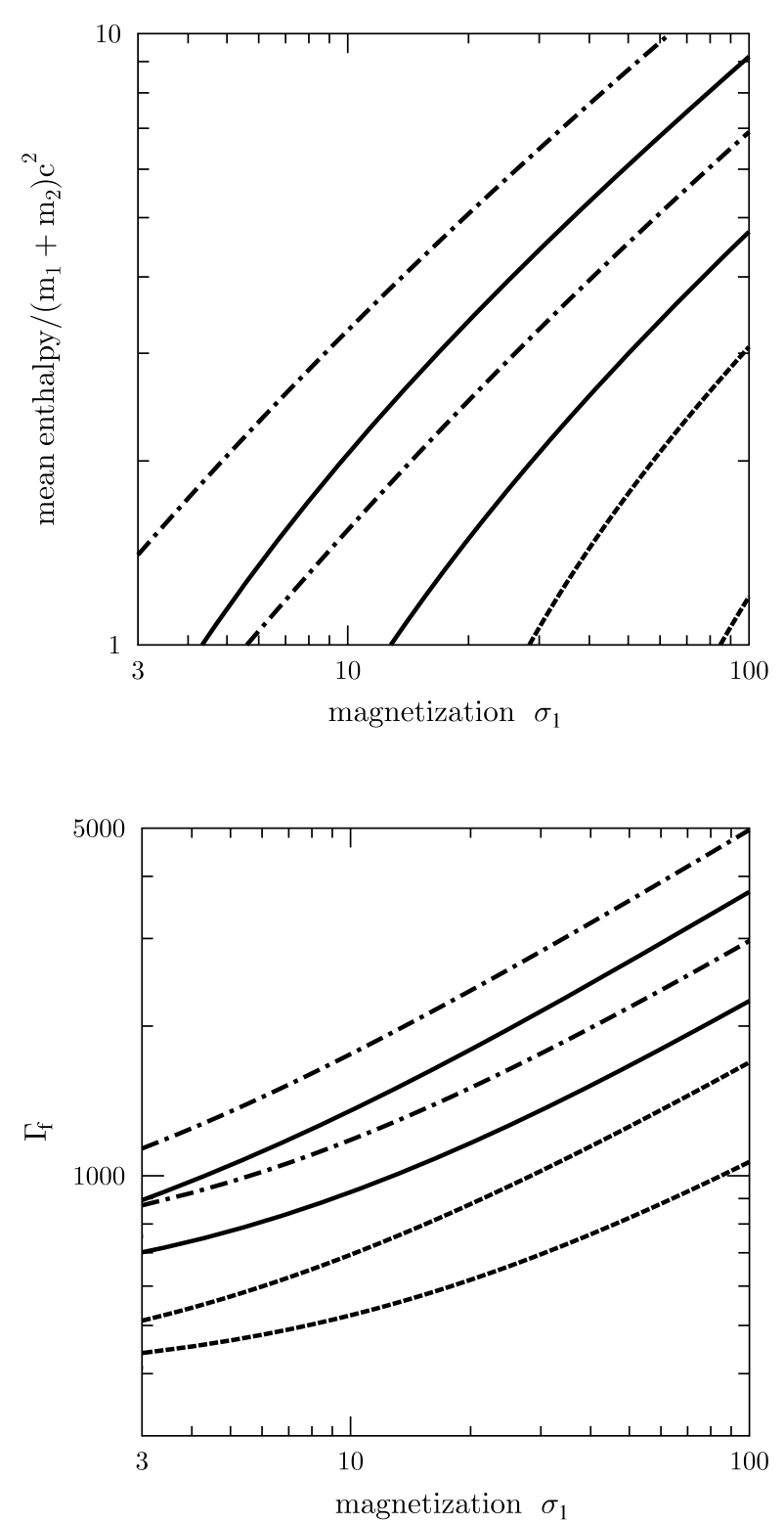

Fig. 4 Simulated Lorentz factor $\Gamma_{\mathrm{f}}$ of a hot merged shell in the observer frame. The curves are simulated for the same parameter sets as it is indicated in Fig. 2

from the conservation laws and the equation of state. The adiabatic index is fixed to $\gamma=4 / 3$, though in more accurate numerical simulations it depends on the Lorentz factor $\Gamma_{\mathrm{f}}$. The key parameter here is the final magnetization of the hot merged shell $\sigma_{\mathrm{f}}$ that is determined by the currently poorly known rate of magnetic field reconnection in the merging shells. The case of fast field reconnection (and, therefore, efficient magnetic field dissipation) is illustrated by the dot-dashed curves in Fig. 2 where $\sigma_{\mathrm{f}}=0.1$. The cases of lower magnetic field dissipation are illustrated by the final magnetization parameters $\sigma_{\mathrm{f}}=1.0$ (solid lines) and $\sigma_{\mathrm{f}}=10.0$ (dashed lines). 
The dependence of the merged shell parameters on the initial magnetization of the colliding shells is presented by two curves of the same style that differ by the initial magnetization parameters. The top curve for each type of line corresponds to colliding shells of equal initial magnetization $\sigma_{1}=\sigma_{2}$. The lower curves of each type correspond to the case when the fast shell of the Lorentz factor $\Gamma_{2}=1000$ has low initial magnetization $\sigma_{2}=0.1$.

It is clearly seen in Fig. 2 that the dissipation efficiency is higher in the case of the initially highly magnetized shells with fast magnetic field reconnection resulted in low $\sigma_{\mathrm{f}}$ of the merged shell (dot-dashed curves). Again, the microscopical model of the reconnection rate in the complex flow is still to be done to estimate the crucial value of the final magnetization parameter $\sigma_{\mathrm{f}}$ of the merged shell (see e.g. Yamada et al. 2010). To investigate the problem of shock formation in the internal dissipation scenario, a microscopic modeling of the collisionless shock formation and its structure in a highly magnetized relativistic outflow is needed, and that is a truly challenging task (see e.g. Bykov and Treumann 2011; Sironi and Spitkovsky 2011). The problem of Fermi acceleration in transverse relativistic shocks of different magnetization that are important to describe the external shocks in the jets of GRBs and AGNs will be addressed in $\S 6$, here we concentrate on the internal dissipation models.

As it is seen in Fig. 4, the high Lorentz factors $\Gamma_{\mathrm{f}}>1000$ of a hot merged shell in the observer frame can be achieved even for the incomplete magnetic field dissipation in the merged shell (solid and dashed curves) and, therefore, it can further catch up other slowly moving shells and merge with them providing a chain dissipation process.

The mean Lorentz factors in Fig. 3 derived from the conservation laws do not preclude a presence of a non-thermal (e.g., a piece-wise power-law) particle distribution, where some minor fraction of particles can reach energies that are by some orders of magnitude larger then the derived "thermodynamic" mean Lorentz factor. Now we turn to discuss in brief possible particle acceleration processes.

\subsection{Particle Acceleration in the Internal Dissipation Models}

Relativistic turbulence produced by the internal dissipation in shocks and magnetic reconnection in relativistic jets result in acceleration processes occurring on both gyro time scale and on longer comoving hydrodynamical time scales of the order of $l / c$. The electric fields induced by turbulent motions of plasmas carrying magnetic fields on different scales lead to statistical energy gains of the superthermal charged particles and their wideband radiation (see Bykov and Meszaros 1996; Mizuno et al. 2011; Nishikawa et al. 2010; Sironi and Spitkovsky 2011; Zhang and Yan 2011; Bykov et al. 2012; Murase et al. 2012).

For nonrelativistic MHD turbulence the particle energy gain over a turbulent correlation length (or correlation time) is small, because the induced electric field is smaller then the entrained magnetic field. However, the distinctive feature of statistical acceleration in the relativistic MHD turbulence and shocks on larger scales expected in the flow-colliding regions, is the possibility of a substantial particle energy gain over one correlation scale, because the induced electric fields are no longer small. In this case the standard Fokker-Planck approach cannot be used. Instead, it is possible to calculate the energy spectra of nonthermal particles within a special integro-differential equation which is a generalization of the Fokker-Planck approach (see Bykov and Toptygin 1993; Bykov and Meszaros 1996).

Charged particles interact with a wide spectrum of RMHD fields and an internal shock ensemble produced by the colliding shells. In the comoving frame, it is assumed that the fluctuations on all scales up to $\sim \Delta$ (including the internal shock ensemble) are nearly isotropic 
(in the latter case, it is enough if they are forward-backward symmetric). The small mean free path $\lambda$ of the superthermal particles leads to their isotropy in the frame of the local bulk velocity fluctuations. The assumed statistical isotropy of the bulk velocity fluctuations in the comoving frame of the wind results then in a nearly isotropical particle distribution, after averaging over the ensemble of strong fluctuations on scales $\sim l$.

To calculate the spectrum of nonthermal leptons accelerated by an ensemble of internal shocks and large-scale plasma motions in the flow-colliding region (averaged over the statistical ensemble of large-scale field fluctuations) we use a kinetic equation for the nearlyisotropic distribution function $N=\gamma^{2} F$, which takes into account the non-Fokker-Planck behavior of the system (see Bykov and Toptygin 1993; Bykov and Meszaros 1996),

$$
\begin{aligned}
\frac{\partial F(r, \xi, t)}{\partial t}= & Q_{i}(\xi)+\int_{-\infty}^{\infty} \mathrm{d} \xi_{1} D_{1}\left(\xi-\xi_{1}\right) \Delta F\left(r, \xi_{1}, t\right) \\
& +\left(\frac{\partial^{2}}{\partial \xi^{2}}+3 \frac{\partial}{\partial \xi}\right) \int_{-\infty}^{\infty} \mathrm{d} \xi_{1} D_{2}\left(\xi-\xi_{1}\right) F\left(r, \xi_{1}, t\right)
\end{aligned}
$$

Here $\xi=\ln \left(\gamma / \gamma_{i}\right), \gamma_{i}$ is the Lorentz factor of the injected particles, $Q_{i}(\xi) \propto \zeta c n l^{2}$ is the rate of nonthermal particle injection, $n$ is the lepton number density in the local flow comoving frame. The kernels of the integral equation (6) determining the spatial and momentum diffusion are expressed through correlation functions which describe the statistical properties of the large scale MHD turbulence and the shock ensemble. Following the renormalization approach, the Fourier transforms of the kernels $D_{1}^{F}(s)$ and $D_{2}^{F}(s)$ are solutions of a transcendental algebraic system of equations of the form $D_{1,2}^{F}=\Phi_{1,2}\left(D_{1}^{F}, D_{2}^{F}, s\right)$. Here $s$ is a variable which is the Fourier conjugate of $\xi$. Equation (6) and the renormalization equations are valid only for particles with sufficiently small mean free paths $\lambda(\gamma) \ll \Delta$.

It is important to note that the solution of Eq. (6) has a universal behavior, only weakly dependent on the complicated details of the turbulent system. The stationary solution to Eq. (6) with a monoenergetic injection rate $Q_{i}$ has an asymptotical behavior of a power-law form, $N \propto Q_{i} \gamma^{-\mathrm{a}}$, where a $=-0.5+\left[2.25+\theta D_{1}(0) D_{2}^{-1}(0)\right]^{0.5}$, and thus one may take $\theta \sim$ $(l / \Delta)^{2}$. For conditions typical of a developed RMHD turbulence, the ratio of the rate of the scatterings to the acceleration rate is $D_{1}(0) D_{2}^{-1}(0)<1$, and for $\theta<1$ one obtains a $\sim 1$. This hard $\gamma^{-1}$ spectral behavior arises because the acceleration time $\tau_{a} \sim l / c \sim \alpha \Delta / c$ is much shorter than the escape time at the relevant energies, $\tau_{\text {esc }} \sim \Delta^{2} / \kappa \sim \Delta^{2} /(l c) \sim \Delta /(\alpha c)$. The power needed to produce such a spectrum of nonthermal particles increases $\propto \gamma_{\max }$, so it is important to understand its temporal evolution.

In the test particle limit, where the backreaction of the accelerated leptons onto the energy-containing bulk motions is small, we have $N(\gamma, t) \propto \zeta n \gamma^{-1}$ for $\gamma \leq \gamma_{\star}(t)$, where $\gamma_{\star}(t)=\gamma_{i} \exp \left(t / \tau_{a}^{h}\right)$ and

$$
\tau_{a}^{h} \propto l / c \sim \alpha(\Delta / c)
$$

is the typical hydrodynamical acceleration timescale (see, e.g. Bykov and Toptygin 1993), with $\gamma_{i} \sim$ few, $\alpha<1$, and the comoving width of the region energized by shocks equal to $\Delta$. From the energy balance equation, when the value $\gamma_{\star}(t) \sim \gamma_{i} m_{p} / m_{e} \varepsilon \zeta^{-1}$ is reached, the growth must saturate, and the resulting spectrum consists of two branches. One of these is the hard spectrum $N(\gamma) \sim \zeta n \gamma^{-1}$, for $\gamma \leq \gamma_{\star}$, where

$$
\gamma_{\star} \sim \gamma_{i} m_{p} / m_{e} \varepsilon \zeta^{-1}
$$

For the typical scales of the considered problem $\gamma_{i} \sim 1$ and $\zeta \sim 10^{-3}$, so $\gamma_{\star} \sim 10^{5}$ (but it could be even larger since $\varepsilon \sim 1$ for large scale plasma motions). In the regime of 
very efficient particle acceleration where the backreaction of the accelerated leptons on the energy-containing bulk motions is substantial, one should use a non-linear approach. In that case the particle distribution experience fast temporal evolution (see e.g. Bykov 2001; Ferrand and Marcowith 2010).

The multiwavelength observations discussed above can be used to constrain the characteristics of relativistic turbulence and particle spectra evolution. Measurements of polarization of prompt gamma-ray emission of GRBs would be very informative. Polarization measurements provide an additional deep insight into the nature of acceleration/radiation processes. By now only a few instruments are available for this kind of measurement at gammaray energies. Gamma-ray bursts (GRBs) are very promising candidates for polarimetry due to their large flux over limited time intervals, maximizing the available signal-to-noise ratio. To date, a few polarization measurements have been reported, all claiming a high degree of polarization in the prompt emission of GRBs, but with rather low statistical evidence yet. Götz et al. (2009) used the IBIS telescope aboard the INTEGRAL space laboratory to measure the polarization of the prompt gamma-ray emission of the long and bright GRB 041219A in the $200-800 \mathrm{keV}$ energy band. They found a variable degree of polarization ranging from less than $4 \%$ over the first peak to $43 \%-25 \%$ for the whole second peak. Time-resolved analysis of both peaks indicated a high degree of polarization, and the small average polarization in the first peak can be explained by the rapid variations observed in the polarization angle and degree. The results by Götz et al. (2009) are consistent with different models for the prompt emission of GRBs at these energies, but they favor synchrotron radiation from a relativistic outflow with a magnetic field, which is coherent on an angular size comparable with the angular size of the emitting region. Recently, Yonetoku et al. (2011) reported the polarization measurement of the prompt gamma-ray emission of GRB 100826A with the Gamma-Ray Burst Polarimeter (GAP) aboard the small solar-sail demonstrator IKAROS. They detected the change of polarization angle during the prompt emission, and the average polarization degree of $27 \pm 11 \%$. Polarization measurements are a powerful tool to constrain the GRB radiation mechanisms and the magnetic field structure in the photon emitting regions.

\section{Active Galactic Nuclei}

\subsection{Studies of Particle Acceleration in AGN Jets}

The relativistic outflows of Active Galactic Nuclei (AGNs) are well-studied particle accelerators. The AGN jet phenomenon spans many orders of magnitudes: jets originate on sub-pc scales close to supermassive black holes with typical masses between a few million and a few billion solar masses and can propagate over many hundred kpc to feed giant hot-spot complexes and radio lobes. One of the most remarkable properties of jets is that they dissipate little energy while transporting vast amounts of energy and momentum over large distances. However, jets are not dissipation-free neither one sub-pc scales nor on kpc-scales: they do accelerate electrons and maybe also protons to high energies. The high-energy particles interact with magnetic fields, photon fields, and/or ambient matter and make AGNs some of the brightest extragalactic sources of continuum emission across the electromagnetic spectrum. There are many reasons to study particle acceleration in AGNs: we would like to explain the observed electromagnetic radiation in order to constrain the composition and structure of jets. The composition and structure constrain the processes of AGN accretion and jet formation, acceleration and collimation. The same studies can enhance 
our understanding of AGN feedback, i.e. how AGNs interact with their hosts and decisively impact their evolution. Studies of AGNs also allow us to study the particle acceleration mechanisms. A good ("microscopic") understanding of the dissipation processes in jets is instrumental for addressing the larger questions mentioned before.

In the following we discuss two particle acceleration sites: the "blazar zone" less than a pc away from the supermassive black hole, and jet particle acceleration by kpc-scale jets. The processes at work in these two well-separated regions have recently received a lot of attention mainly because of spectacular jet images from the VLBA, HST, and Chandra, and spectacular multiwavelength observations with the RXTE, Suzaku, and Swift, X-ray, and Fermi, H.E.S.S., MAGIC, and VERITAS $\gamma$-ray telescopes.

\subsection{Studies of Particle Acceleration Processes in the Blazar Zone}

The cores of the "blazar class" of AGNs are bright sources of spatially unresolved continuum emission. In the case of BL Lac objects, emission and absorption lines are absent or weak. In the case of their more powerful siblings, Flat Spectrum Radio Quasars (FSRQs), the spectra exhibit emission and absorption lines, which afford additional diagnostics concerning the mass of the central black hole, the accretion power, and the redshift of the source. Blazars are sources with jets closely aligned with the line of sight. The relativistic propagation of the jet plasma and the close alignment of the jet with the line of sight lead to relativistic boosting of the emission. Blazar Spectral Energy Distributions (SEDs) show evidence for two broad peaks presumably coming from synchrotron and inverse Compton emission from the same electron population. Observations of the BL Lac Mrk 501 revealed emission up to $16 \mathrm{TeV}$ (Aharonian et al. 1999), giving direct proof that AGN jets accelerate particles to $\gg 1 \mathrm{TeV}$ energies. AGNs may accelerate particles to much higher energies, maybe even to ultra-high energies (e.g. Kachelrießet al. 2010), see however Lemoine and Waxman (2009) for a detailed discussion of acceleration power in these sources.

Two scenarios are commonly invoked to explain the observed emission: (i) the jet is initially Poynting flux dominated and accelerates particles in magnetic reconnection events (e.g. Giannios et al. 2010, and references therein); (ii) the jet is either particle energy dominated right from the start, or, it is initially Poynting flux dominated and some unknown mechanism converts the energy from Poynting flux into bulk motion energy, and the particle dominated jets subsequently accelerate particles at shocks.

Blazar studies of particle acceleration benefit from the fact that the broad-band SEDs oft he sources evolve on short time scales: X-ray and gamma-ray flares with 1 min durations have been reported. It thus is possible to track the temporal evolution of the particle energy spectra. Modeling of sequences of broad-band SEDs has shown that the jets are highly relativistic with bulk Lorentz factors of $\sim 50$ or even higher (e.g. Krawczynski et al. 2001). The simplest types of models synchrotron self-Compton models-indicate that the particle energy density dominates strongly over the magnetic field energy density in the blazar zone (e.g. Krawczynski et al. 2002; Acciari et al. 2011). These results clearly favor shock acceleration over magnetic reconnection. The data and theoretical studies (particlein-cell (PIC) simulations and instability analyses) favor mildly relativistic shocks internal to the jets over highly relativistic external shocks: the former have faster downstream plasmas commensurate with the high inferred bulk Lorentz factors of the emitting plasma, and are less susceptible to the strong suppression of the formation of upstream scattering centers by even a weak plasma magnetization than their highly relativistic counterparts (e.g. Lemoine and Pelletier 2010; Sironi and Spitkovsky 2009).

$\mathrm{X}$-ray and very high-energy $\gamma$-ray observatories have recently succeeded to sample the temporal evolution of the X-ray and X-ray energy spectra with delicate accuracy. Although 
observers organized a large number of observation campaigns with the objective to find the flux vs. spectral index evolution patterns predicted by simple acceleration theory (e.g. Kirk et al. 1998), the observations revealed rather erratic evolutions (e.g. Takahashi et al. 2000; Garson et al. 2010, and references therein). Unfortunately, observations have not yet succeeded to determine unambiguously where exactly the blazar emission originates. For the radio galaxy M87 possibly a misaligned blazar the observation of temporally coincident radio, X-ray, and $\gamma$-ray flare indicates that the emission comes from $<100$ Schwarzschild radii of the supermassive black hole (Acciari et al. 2009).

\subsection{Studies of Particle Acceleration Processes of kpc-scale Radio, Optical, and X-Ray Jets}

The VLBA, HST, and Chandra telescopes are delivering images of the kpc-jets of an ever increasing number of radio galaxies. The images in the radio, optical, and X-ray bands can be used to infer complementary information about the emitting particle populations. Radio and optical polarimetry give additional clues about the orientation of the magnetic field in the bright jet regions.

A recent somewhat surprising result was the detection of a large number of $\mathrm{X}$-ray bright kpc-jets with Chandra (see the review by Harris and Krawczynski 2006, and references therein). In the case of powerful Fanaroff-Riley (FR) type II sources (like PKS 0637-752) the combined radio, optical and X-ray energy spectra showed unambiguous evidence for two distinct emission components. Presently two models are commonly invoked to explain the "second" X-ray bright component: (i) inverse Compton scattering of Cosmic Microwave Background photons (Tavecchio et al. 2000; Celotti et al. 2001), (ii) a second synchrotron component (Harris and Krawczynski 2002). The first model requires jet plasma moving with large bulk Lorentz factors $(\Gamma \sim 10)$ at kpc-distances from the central engine. The relativistic motion boosts the CMB photon energy density in the reference frame of the emitting plasma by a factor of $\Gamma^{2}$, and the mean photon energy by a factor of $\Gamma$. These two effects make it possible to explain the observed X-ray emission with electrons with modest electron Lorentz factors on the order of $\gamma=100$. The second model requires much higher Lorentz factors: assuming equipartition magnetic fields, electrons with Lorentz factors $\gamma \sim 10^{7}$ are needed to explain the X-ray emission. Both models have severe shortcomings. For example, in the first model the long cooling times of the low- $\gamma$ electrons are at odds with the well-defined knotty structure of some X-ray bright jets. A weakness of the second model is that there is not yet a good explanation for the existence of two distinct electron populations with very different spectral properties. For lower-power FR-I-type sources like M87, Cen A, or 3C 31, the combined radio, optical and X-ray energy spectra are consistent with a synchrotron-origin of the $\mathrm{X}$-ray emission from a single population of electrons. For an equipartition magnetic fields of $B \sim 100 \mu \mathrm{G}$, the X-ray emitting electrons have Lorentz factors $\gamma \sim 10^{7}$, and radiative synchrotron cooling times of a few years. The X-ray emitting electrons thus do not move far from their acceleration sites before they loose their energy, and the X-ray brightness profiles tracks the acceleration of the high-energy electrons. The diffuse appearance of some jets implies quasi-continuous acceleration.

A few radio galaxy jets have been studied with the Hubble Space Telescope giving not only high-resolution images of the optical brightness but also of the optical polarization. The magnetic field probed by the optically emitting electrons seems to be aligned parallel to the jet flow for the most part. However, upstream of the brightness maxima, the field are perpendicular to the jet flow. The radio and optical polarization behavior differs, indicating that the emission at different wavelengths samples different regions of the jet (Perlman et al. 1999). 


\section{Acceleration to Ultra-high-Energies}

\subsection{Some Properties of UHECRs}

One of the 11 fundamental science questions for the 21 st century listed in the final report of the 2002 Decadal Review (Turner et al. 2002) is the nature of cosmic rays. The detection of cosmic rays at ultra-high energies (UHECR) dates back to the early Sixties, but only during the last 20 years detectors of sufficiently large size have become operational, that the origin of UHECRs can be addressed (for a review see Kotera and Olinto 2011). UHECRs are indirectly detected by observing the airshowers they trigger in the atmosphere. One can look for fluorescence emission or other radiation produced high in the atmosphere, or, alternatively, one registers the passage of secondary particle in charged-particle detectors on the ground. The AUGER observatory (Abraham et al. 2004) and the Telescope Array (Kawai et al. 2008) combine both techniques.

The main observables used to infer the properties of UHECRs are the anisotropy, the composition, and the spectrum. The composition is difficult to determine, because the appearance of giant airshowers can only be modeled with particle-physics event generators that involve extrapolations of behaviour observed in accelerator experiments, for the CoM energy of an arbitrary nucleus of $10 \mathrm{EeV}$ energy with a nitrogen nucleus at rest is far higher than that achievable with even the largest man-made accelerator, the LHC at CERN. Considerable systematic uncertainty thus overshadows attempts to study the composition. It appears that between $1 \mathrm{PeV}$ and $0.1 \mathrm{EeV}$ we observe a trend from a predominance of light particles to heavy nuclei. Around $1 \mathrm{EeV}$, the composition is light again (Abbasi et al. 2010; Abraham et al. 2010b). Above $1 \mathrm{EeV}$, Auger observes a transition to heavier particles that is not see with other experiments at this time, possibly on account of statistics.

The anisotropy is low around $1 \mathrm{EeV}$, where upper limits near $1 \%$ have been published for the sidereal dipole anisotropy (Abreu et al. 2011). At higher energies above $57 \mathrm{EeV}$, for which little deflection would be expected, if the primary particles were protons, a correlation is observed between the arrival direction of particles and certain types of nearby AGN which in the end are proxies of the matter distribution within $\sim 75 \mathrm{Mpc}$ from us (Abraham et al. 2008; Abreu et al. 2010). The distance limitation is expected because nuclei at these energies undergo photodisintegration and photomeson production that provide losses on corresponding time scales, leading to the so-called GZK cut off.

Whereas cosmic rays approximately obey a power-law spectrum with index $s \simeq 2.7$ $\left(d N / d E \propto E^{-s}\right)$ below the so-called knee in the spectrum at $3 \mathrm{PeV}$, the spectrum of UHECRs is soft between a few PeV and $3 \mathrm{EeV}$ with a power-law index $s \simeq 3$ (Apel et al. 2009). At $3 \mathrm{EeV}$ the spectrum hardens to $s \simeq 2.6$, a feature known as the ankle. Above $30 \mathrm{EeV}$ one observes a flux suppression that has been identified with the GZK cut off (Abraham et al. 2010a) A recent proposal (Aloisio et al. 2011) interprets those features as a proton cut off around $10^{18} \mathrm{eV}$ and another one around $3 \times 10^{19} \mathrm{eV}$ associated with iron nuclei. ${ }^{1}$

\subsection{Implications of the Maximum Energy}

Cosmic rays at energies below $1 \mathrm{PeV}$ are almost certainly galactic in origin, and those at energies above $10 \mathrm{EeV}$ are most likely extragalactic, but considerable uncertainty exists at intermediate energies. It is unclear at what energy the local cosmic rays turn from being

\footnotetext{
${ }^{1}$ This scenario implicitly postulates a very large proton-to-helium ratio in the source.
} 
predominantly galactic to being mostly extragalactic. The relevance of this uncertainty for modeling the sources of cosmic rays is obvious: if the particles in the energy band above the knee at a few $\mathrm{PeV}$, or above the iron knee at $\sim 10^{17} \mathrm{eV}$, are extragalactic, then considerable finetuning is required in matching the galactic and extragalactic components, because the spectrum softens at the knee. On the other hand, if cosmic rays up to a few EeV, i.e. up to the ankle in the spectrum, are galactic, then no such finetuning is required, but we need to identify the sources of EeV-band cosmic rays with objects present in the Galaxy, e.g. supernova remnants (SNR), pulsars, etc. This can be difficult, not because the source in question would not accelerate particles to high energies, which in fact we observe happening in SNR and pulsars, but because it is questionable that EeV energies can be reached.

In fact, for typical interstellar magnetic field values, SNR shock fronts can hardly accelerate cosmic rays to a PeV (Lagage and Cesarsky 1983a, 1983b). Particle confinement near the shock is supported by self-generated magnetic turbulence ahead of and behind the shock. Various plasma instabilities driven by cosmic rays can contribute to excite the turbulence to high levels, although which dominates remains an active topic of research. In the case of SNRs, that which has received most attention so far is the so-called streaming instability seeded by the cosmic-ray net current (e.g. Wentzel 1974; Skilling 1975; Achterberg 1983; Lucek and Bell 2000; Bell and Lucek 2001), and more recently its non-resonant counterpart (Bell 2004, 2005; Pelletier et al. 2006). In contrast, relativistic shocks operating at the interface between AGN/GRB flows and the surrounding medium reveal a short precursor, which restricts the plasma instabilities to small scale modes (Medvedev and Loeb 1999; Pohl and Schlickeiser 2000; Pohl et al. 2002; Reville et al. 2006; Pelletier et al. 2009; Lemoine and Pelletier 2010, 2011a), as discussed in detail in the following. Clearly, the amplitude of the turbulence sets up the pitch-angle scattering frequency and thus the acceleration rate (Malkov and Diamond 2001). In addition, it also sets the scale for the maximum energy, to which a remnant may accelerate particles. Although analytical and numerical estimates suggest that cosmic rays can very efficiently drive magnetic turbulence ahead of the shock (e.g. Bykov et al. 2011), so the turbulent magnetic field may be much larger than the homogeneous interstellar field (Lucek and Bell 2000; Bell and Lucek 2001), large increases in the magnetic field strength do not necessarily translate into a significant increase in the maximum particle energy (Vladimirov et al. 2006).

For relativistic sources such as AGN or GRB, relativistic shock acceleration can be invoked, but even there certain limitations arise (e.g. Gallant and Achterberg 1999; Achterberg et al. 2001; Pelletier et al. 2009; Lemoine and Pelletier 2011b; Eichler and Pohl 2011). To be shock accelerated, a particle that has crossed the shock toward the upstream must be overtaken again by the shock. Assuming the shock moves at Lorentz factor $\Gamma_{S}$, the particle must have been deflected (by gyration or scattering) through an angle $\Delta \theta \gtrsim 1 / \beta_{S} \Gamma_{S}$ while residing upstream. This deflection must be accomplished within a time $\Delta t$ at least of order $R_{S} / \beta_{S} c$, where $R_{S}$ is the shock radius at which the shock once again overtakes the particle. The factor $1 / \beta_{S}$ in $\Delta \theta$ arises for subrelativistic shocks on account of the small incremental energy gain per shock crossing. The particle must cross the shock $\sim 1 / \beta_{S}$ times to double its energy, with each crossing requiring at least a significant fraction of a gyroperiod.

The fastest possible deflection is provided by undisturbed gyration in magnetic field oriented perpendicular to the shock normal, for which the angle between particle momentum and shock normal, $\theta$, increases linearly with time, and the deflection rate $\Delta \theta / \Delta t$ must then obey $\Delta \theta / \Delta t=\beta c / r_{g} \gtrsim c /\left(R_{S} \Gamma_{S}\right)$. This inequality sets a maximum energy $E_{\max }$ to which a particle can be accelerated, because the rate of change of angle presumably decreases with particle energy. As $r_{g}=p c / Z e B=\beta E / Z e B$, this corresponds to a maximum energy, in 
the limit of relativistic particles, of

$$
E_{\max }=Z e B R_{S} \Gamma_{S}
$$

Such regular deflection only occurs in a magnetic field that is coherent over scales larger than the path length of the particle. In the short precursor of a relativistic shock, this restricts $B$ to the background, undisturbed magnetic-field value, and it thus limits the maximal energy to a rather small value for typical interstellar-medium conditions. To be noted is that Eq. (9) may provide a rather academic limit, because in a relativistic shock a perpendicular magnetic field in the downstream region renders acceleration very inefficient. Also note that the magneticfield strength, $B$, is supposed to be measured in the upstream frame of the shock, i.e. it is the ambient field in the source frame in, e.g., an external shock of a GRB.

Note that scatter-free gyration cannot in general confine a CR particle to a subrelativistic blast wave in all three dimensions. The particle generally drifts off to the side after gaining the potential difference $\beta_{S} E B R_{S}$ in energy. Some scattering is required which will reduce $E_{\max }$. Also note that we have neglected both adiabatic losses and drift to the periphery of the shock front and assumed that being overtaken by a spherical blast wave is sufficient for further acceleration.

For relativistic shocks, escape through the lateral boundaries does not provide a stringent constraint on the maximal acceleration energy unless sideways expansion of the blast takes place: as viewed in the shock front rest frame, the particle is confined if its gyration radius $r_{g, 0 \mid \mathrm{sh}}<R_{\perp}$, with $R_{\perp}$ the lateral extension of the shock front. Since $r_{g, 0 \mid \mathrm{sh}} \simeq r_{g, 0} / \Gamma_{S}^{2}$, with $r_{g}$ the upstream gyroradius in the background field, confinement leads to $E_{\max }<\Gamma_{S}^{2} R_{\perp} e B$, which is not as restrictive as the previous expression if $R_{\perp}>R_{S} / \Gamma_{S}$. In that limit, sideways expansion of the blast is negligible and the overall dynamics resembles that of a spherical blast wave.

What of often invoked magnetic-field amplification by cosmic-ray induced instabilities? The growth of plasma instabilities in the precursors of shocks is inevitable, and therefore in a realistic situation we cannot expect undisturbed gyration in perpendicular magnetic-field. In fact, random scattering is required if the large-scale magnetic field is oriented parallel to the shock, because otherwise particles could not return to the shock. Therefore, random scattering in small-scale fields will make acceleration at parallel shocks faster, and thereby increase the maximum energy, in particular at nonrelativistic shocks.

The scattering mean free path can be written as $\lambda \sim c / D_{\theta \theta}$, where the angular diffusion coefficient is given by $D_{\theta \theta}=\delta \theta^{2} / \delta t \sim\left(e B_{\text {rms }} / \beta \Gamma m c\right)^{2} l / \beta c$ where $\delta t \sim l / \beta c$ is the scattering coherence time, over which the particle scatters by an angle $\delta \theta$, and $l$ is the coherence length of the magnetic field (e.g. Eichler and Pohl 2011; Plotnikov et al. 2011). At sub-relativistic shock waves, one must now impose $r_{g}^{2} / l \leq \beta_{S} R_{S}$, with $r_{g}=p c / Z e B_{\mathrm{rms}}$. For relativistic shocks, the condition for the particle to suffer a rms deflection $1 / \Gamma_{S}$ over a timescale $R_{S} / c$ reads $r_{g}^{2} / l \leq \Gamma_{S}^{2} R_{S}$, so that these two equations can be combined into

$$
E_{\max } \leq Z e B_{\mathrm{rms}}\left(\beta_{S} l R_{S}\right)^{\frac{1}{2}} \Gamma_{S}
$$

which is less than the previous expression when $l \ll r_{g}$. Such small-scale fields are expected in the precursor of relativistic shocks, which cannot exceed $r_{g, 0} / \Gamma_{s}^{3}$, although there is then an ambiguity related to the reference frame of the small-scale magnetic inhomogeneities; for simplicity, we have assumed here that these magnetic inhomogeneities are at rest in the upstream plasma. Thus, provided some large-scale perpendicular magnetic field exists, simply tangling the field on small scales, $l$, does not necessarily raise $E_{\max }$. Magnetic-field amplification enhances the maximum energy only if it increases $B_{\mathrm{rms}}^{2} l$. Note that the expression 
$E_{\max }=Z e B R_{S}$, often taken from Fig. 1 of Hillas (1984), is consistent with Eqs. (9) or (10) only if $\beta_{s}$ and $\Gamma_{s}$ are both of order unity. We stress again that the limits described here may not be reached at a real shock. Leakage from the precursor and the conditions downstream must also be considered when evaluating the maximum energy and the acceleration efficiency.

Equations (9) and (10) suggest that the sources of UHECRs are likely systems involving relativistic shocks.

\subsection{Sources of UHECRs}

Besides reaching the required particle energy, the sources of UHECR must also be powerful enough to provide the source luminosity needed to sustain the local flux of UHECR. Possible source candidates of UHECRs are active galactic nuclei (AGNs) (Biermann and Strittmatter 1987; Takahara 1990; Rachen and Biermann 1993; Pe'Er et al. 2009), clusters of galaxies (Kang et al. 1997), Magnetars (Arons 2003; Murase et al. 2009), and gamma-ray bursts (GRBs) (Waxman 1995; Vietri 1995; Murase et al. 2006). Depending on the model, these sources may also dominate the energy range around $10^{18} \mathrm{eV}$ (Berezinsky et al. 2006; Wang et al. 2007; Murase et al. 2008).

One can constrain the acceleration capabilities of various sources through the magnetic luminosity of these sources (e.g. Norman et al. 1995; Waxman 2005; Lemoine and Waxman 2009), as follows. Let us assume that acceleration takes place in an outflow at radius $r$ moving with possibly relativistic velocity $\beta$ (and Lorentz factor $\Gamma$ ) towards the observer, so as to benefit from Lorentz boosting. We assume that acceleration proceeds with an acceleration timescale $t_{\text {acc }} \equiv \mathcal{A} r_{g} / c$ in the comoving frame, with $\mathcal{A}>1$. Then the maximal energy at acceleration is at least bounded by the condition $t_{\text {acc }}<r /(\Gamma \beta c)$, which means that the acceleration timescale must be shorter than the comoving age of the outflow. This limit can be rewritten in terms of the maximal energy in the observer frame, $E_{\max }$ and in terms of the magnetic luminosity of the source, $L_{B} \equiv r^{2} \Theta^{2} \Gamma^{2} \beta c B^{2} / 4$ as calculated in the source rest frame in terms of the jet half opening angle $\Theta$ and comoving magnetic-field strength $B$ :

$$
E_{\max } \lesssim 10^{20} \mathrm{eV}^{-1} \Gamma^{-1} \Theta^{-1} \beta^{-3 / 2} Z L_{B, 45}^{1 / 2}
$$

with $L_{B, 45}=L_{B} / 10^{45} \mathrm{erg} / \mathrm{s}$. One can check that this bound remains robust in the small $\Theta$ limit, meaning $\Theta \Gamma \rightarrow 0$ for which side escape becomes important, and in the small $\beta$ limit. This bound indicates that rather extraordinary luminosities are required to accelerate particles to ultra-high energies, under rather general conditions, although the bound depends on the charge of the particle. For instance, if one derives the magnetic luminosity of blazars through a leptonic modelling of the spectral energy distributions, one concludes that only the rare flat spectrum radio quasars with jet powers $\gtrsim 10^{44} \ldots 10^{46} \mathrm{erg} / \mathrm{s}$ can accelerate protons to $\sim 10^{20} \mathrm{eV}$, while other Bl Lac and TeV blazars (FR I analogs) with jet powers $\sim 10^{40} \ldots 10^{44} \mathrm{erg} / \mathrm{s}$ appear limited to $\sim 10^{18}-10^{19} \mathrm{eV}$ (Lemoine and Waxman 2009). From this point of view, more compact sources such as GRBs and magnetars appear favored. For instance, a GRB of apparent isotropic luminosity $10^{52} \mathrm{erg} / \mathrm{s}$ with $\Gamma \sim 100$ may produce particles with energy as high as $Z \times 10^{21} \mathrm{eV}$ for a magnetic conversion factor $\xi_{B}=0.01$. As discussed in Sect. 6.3, mildly or sub-relativistic shocks in a relativistic flow are more efficient accelerators of protons than ultra-relativistic shocks and are excellent candidates for being sources of UHECRs, owing to the magnetic-field amplification at shocks.

The paucity of FR 2 radio-galaxies in the GZK sphere (radius $\sim 100 \mathrm{Mpc}$ ) capable of accelerating protons to ultra-high energies might be compensated by the acceleration of 
heavier nuclei in the less powerful and more numerous FR I radio-galaxies. In particular, Ptuskin and collaborators have shown that if radio-galaxies inject of light to heavy elements with a rigidity dependent maximal energy following Eq. (11), $L_{B}$ being related to the radio luminosity, accounting for the radio-luminosity function, one could explain rather satisfactorily the observed spectrum (Ptuskin et al. 2011). It is also intriguing that the Pierre Auger Observatory reports an excess of events in the direction of the nearby radio-galaxy Cen A (although this latter happens to lie in front of one of the largest concentrations of matter in the GZK sphere, the Centaurus supercluster). However, it would be very difficult to understand the observed pattern of anisotropy if one assumes that the highest energy particles are heavier than hydrogen in such scenarios (Lemoine and Waxman 2009).

Besides the actual source physics, the evolution of sources, the number of accelerators within a source (Aloisio et al. 2007), and the variation of source properties (Kachelriess and Semikoz 2006; Berezhko 2008) will also shape the local spectrum of UHECRs.

Many properties of UHECRs can be impacted by their propagation in intergalactic space, such as their composition through photo-disintegration or their spectrum through cascading via photo-meson and photo-pair production, but it is difficult to disentangle the propagation effects from the results of physical processes operating inside the sources of these particles.

Estimating the source luminosity using observed quantities and the known population statistics of the sources in question is subject to considerable uncertainties. As an example, Eichler et al. (2010) have recently estimated the local UHECR source luminosity, assuming all particles above the ankle at $4 \mathrm{EeV}$ are extragalactic, and compared that with the observed gamma-ray production rate of all GRB. In contrast to earlier studies (Waxman 2004; Le and Dermer 2007), not the MeV-band gamma-ray fluence was used, which likely represents a thermal pool, but the GeV-band emission observed with Fermi-LAT, which measures the non-thermal tail of the energy distribution in the GRB primary charged particles, which, if hadronic, is the part that could contribute to the UHECR flux. It turns out that the UHECR source luminosity is more than a hundred times higher than the total GeV-band photon output, which places severe constraints on UHECR models involving GRB. If one posits that the Galactic to extra-Galactic transition takes place at $\sim 10^{19} \mathrm{eV}$ and the MeV gammaray fluence traces the nonthermal particle population, the particle output of GRBs is more commensurate with their photon output (Waxman 2010).

For each source class, one can also estimate a luminosity function, that is the differential source density needed to integrate the contribution of the sources over cosmological redshift. While only nearby $(\lesssim 200 \mathrm{Mpc})$ sources may actually contribute to locally observable GZK-scale UHECRs, the interaction products of the particles from all more distant sources will feed a cascade of energy that is eventually observable as a component of the extragalactic gamma-ray and neutrino background, which are two other cosmic messengers that are complementary to the charged particles. The former has been recently measured with unprecedented sensitivity up to $100 \mathrm{GeV}$ (Abdo et al. 2010), and thus provides invaluable constraints on, e.g., the so-called dip models, which assume that essentially all particle above about $1 \mathrm{EeV}$ are protons. The redshift of the onset of photopair production with the CMB would then naturally lead to an ankle at the energy where it is indeed observed (Berezinsky et al. 2006). More precisely, it is the cosmic evolution of the source class in question that determines how much energy is fed into an electromagnetic cascade and eventually reappears in the GeV-band background radiation, relative to the UHECR energy flux at the ankle.

\subsection{The Transition from Galactic to Extragalactic Origin}

An open problem in cosmic-ray astrophysics is at what energy we observe the transition from a Galactic to an extragalactic origin of particles. The limit on inferred source power 
per unit baryon mass required to sustain Galactic UHECR in the [4-40] EeV range that is imposed by the observed anisotropy limits is smaller by nearly 3 orders of magnitude than what is required for an extragalactic origin, as calculated in Eichler et al. (2010), and it corresponds to the power per unit mass of gamma rays from GRB (Eichler and Pohl 2011). This is not only confirmation of the hypothesis that UHECR beyond the ankle are extragalactic, it also suggest that their sources are systems not persistently present in the Galaxy. Any astrophysical source class, that is capable of accelerating particles to very high energies and should exist in the Galaxy, may fall short of accounting for the trans-ankle UHECR, but may nevertheless significantly contribute to the observed cosmic-ray flux between the knee and the ankle. For example, the numerical coincidence fits the hypothesis of a GRB origin for the Galactic component of UHECR, without invoking a much larger unseen energy reservoir for GRB.

The interesting question is the rate with which such sources appear in normal galaxies such as the Milky Way. In other words, what is the role of intermittency? Generally, GRBs in the Galaxy are expected every million years or so, the exact rate depending on the beaming fraction and the detailed scaling of long GRB with star formation and metallicity. Therefore, only a small number of GRB can contribute to the particle flux at the solar circle, and their relative contribution depends on the location and explosion time of the GRB. Variations in the local particle flux must be expected, and neither the particle spectrum from an individual GRB nor the spectrum calculated for a homogeneous source distribution are good proxies.

Pohl and Eichler (2011) have calculated the time-dependent transport of UHECR in the Galaxy, assuming it can be described as isotropic diffusion. They find that intermittency becomes serious if the mean free path for scattering exceeds $100 \mathrm{pc}$, unless the source rate is much higher than 1 per Myr. On average, Galactic long GRB need to contribute only about $10^{37} \mathrm{erg} / \mathrm{s}$ in accelerated particles to fully account for the observed particle flux at $10^{18} \mathrm{eV}$, assuming a Bohmian mean free path at this energy. UHECR from Galactic long GRB can meet the observational limits on anisotropy only if the mean free path for scattering is sufficiently small. Contributing the observed sub-ankle particles (at $10^{18} \mathrm{eV}$ ) requires Bohmian diffusion if the UHECR are as heavy as carbon. A light composition such as protons or helium requires sub-Bohmian diffusion, which is a highly unlikely situation for isotropic diffusion.

Much of the UHECR anisotropy arises from the expected location of long GRB in the inner Galaxy. Observations of GRB host galaxies suggest that regions of low metallicity and active star formation may be the preferred sites of long GRB (Levesque et al. 2010; Levesque 2011), which may skew the galactocentric distribution of long GRB toward the outer Galaxy. As there is no power problem with Galactic GRB, it may be worthwhile to also consider short GRB. They provide supposedly less power as a population, but they may have a very extended spatial distribution in the Galaxy (Berger 2010), leading to a reduced, but on account of intermittency not disappearing anisotropy.

These conclusions can be applied with little change to the case of an origin of UHECRs in SNRs, assuming very efficient magnetic-field amplification can increase their ability to accelerate particles to energies significantly higher than $1 \mathrm{PeV}$ (e.g. Ptuskin et al. 2010). The spatial distribution in the Galaxy of long GRB and SNR can be expected to be similar, and therefore the average anisotropy is the same for both long GRB and SNR. If one combines such a galactic component with a dip model, so that the galactic/extragalactic transition occurs below $1 \mathrm{EeV}$, Bohm diffusion and a mixed composition of the Galactic component may still be viable, given the systematic uncertainties in the measurements. It would be highly desirable to improve anisotropy measurement between $0.1 \mathrm{EeV}$ and $1 \mathrm{EeV}$, and likewise better constrain the composition. 


\subsection{UHECR Summary}

Recent progress in UHECR research has built on data from new large-scale observatories. The interpretation of measurements of the composition, anisotropy, and spectrum of particles provides constraining links between these observables, that are further strengthened by new precision measurement of, e.g., extragalactic gamma-ray background emission. The very low anisotropy observed for EeV-scale particles provides a strong limit on the contribution of Galactic sources, if the composition is indeed light as suggested by data. The anisotropy found above $60 \mathrm{EeV}$ would be difficult to understand if the particles were heavy, which is suggested by Auger data, but not HiRes. If these particles were light, dip models might be favorable which, however, must be carefully constructed to not overproduce the $50-\mathrm{GeV}$-scale gamma-ray background.

The main obstacle to further progress clearly is the systematic uncertainty arising from the interpretation of the evolution of giant airshowers with particle-physics models that are extrapolated over at least 1.5 decades in CoM energy from the range testable with manmade accelerator experiments.

\section{Particle Acceleration at Relativistic Shocks}

Strong shocks occurring in astrophysical flows often generate power-law distributions of very-high-energy particles. This is the origin of most high-energy phenomena in astrophysics. The favored mechanism for the generation of supra-thermal particles is the famous Fermi process. It involves with the scattering of high-energy particles off magnetic disturbances that allow them to cross the shock back and forth and thus to gain energy. Many studies have been performed in the $80 \mathrm{~s}$ and $90 \mathrm{~s}$ by assuming pre-existing magnetic turbulence. However, it turns out that the pre-existing turbulence is generally not strong enough to account for the acceleration performance. The nonthermal X-ray emission from SNRs (Cassam-Chenaï et al. 2004), but see also Pohl et al. (2005), revealed that the magnetic field is strongly amplified in the vicinity of the forward shock. Recent theoretical studies have shown that the penetration of accelerated particles in the shock upstream flow can generate magnetic turbulence that reaches a level much larger than the intensity of the ambient mean field (Bell 2004; Pelletier et al. 2006). In producing turbulence the cosmic rays loose a fraction of its global energy (about 10 percent of the incoming energy) but increases the maximum energy of particles (cf. Sect. 5.2). The turbulent field can reach an intensity of a few hundreds of $\mu \mathrm{G}$, much larger than the value of a few $\mu \mathrm{G}$ of the ambient magnetic field in the Galaxy.

These results incited similar investigations for relativistic shocks. Very encouraging results were obtained around the turn of the century which extended the theory of Fermi process to the case of relativistic shocks and predicted the formation of a power-law energy spectrum with an index $s=2.2-2.3$ and an acceleration time as fast as the Larmor time (Bednarz and Ostrowski 1998; Gallant and Achterberg 1999; Kirk et al. 2000; Achterberg et al. 2001; Ellison and Double 2002; Lemoine and Pelletier 2003). But disappointment came once the effect of the ambient magnetic field had been taken into account, because it inhibits the Fermi process even when one considers a strong Kolmogorov turbulence (Niemiec et al. 2006; Lemoine et al. 2006).

In the following, it will be shown how the paradigm of the three interdependent aspects of collisionless-shock physics successfully works in the absence of any mean field: structure with a partial reflection on a barrier, supra-thermal-particle generation, magnetic-turbulence 
generation. Then the scattering issue in the presence of a mean magnetic field will be addressed and the requirement for circumventing the inhibition effect will be stated. Then an unusual fact in astrophysics will be emphasized, namely the necessity of considering some unavoidable micro-physics, that turns out to be crucial not only for the relativistic shock formation but also for making the Fermi process operative and producing high energy particles.

\subsection{Successful Fermi Process at Very Low Magnetization}

The most favored process for the generation of supra-thermal power law distributions is the Fermi process at shocks. Under astrophysical conditions the plasma flow that experiences a shock is supposed to carry a frozen-in turbulent magnetic field which allows particle scattering, and thereby permits particles to gain energy at each Fermi cycle, i.e. a cycle upstream-downstream-upstream or downstream-upstream-downstream.

At a non-relativistic shock of speed $\beta_{s}=V_{s} / c \ll 1$, the average gain per cycle is small, $G=1+\frac{4}{3} \frac{r-1}{r} \beta_{s}$ (where $r$ is the compression ratio, that reaches the value 4 when the shock is adiabatic and strong). However this is compensated by a large number of shock crossings; indeed the escape probability (i.e. the probability for a particle to be entrained by the downstream flow and to not come back to the shock front) is low, $P_{\text {esc }}=4 \beta_{s} / r$; the return probability $P_{\text {ret }}$ is thus large. A power-law distribution of energy is set up with an index that is a simple function of the compression ratio, in the non-relativistic case:

$$
s=1-\frac{\ln P_{\text {ret }}}{\ln G} \simeq 1+\frac{3}{r-1} .
$$

Strong adiabatic shocks provide a particle spectrum with an universal index, $s \simeq 2$, which is modified by losses, radiation losses for the electrons, expansion or escape for protons. Subsequent to escape, the spectrum is then steepened by the effect of diffusive propagation and escape of particles from the Galaxy.

A sizable fraction of the incoming energy flux is converted into cosmic ray pressure:

$$
P_{\mathrm{cr}}=\xi_{\mathrm{cr}} \rho_{u} V_{s}^{2} \quad \text { with } \xi_{\mathrm{cr}} \sim 0.1
$$

The successive Fermi cycles produce a precursor of supra-thermal particles (mostly protons) of large extension (the diffusion length increases with the particle energy) and this penetration in the upstream medium (the ambient medium for an external shock) triggers MHD turbulence through two types of streaming instability, one is resonant and has been considered for many years (see for instance McKenzie and Voelk 1982), the other is nonresonant and has been considered more recently (Bell 2004; Pelletier et al. 2006), as briefly discussed earlier. That latter case is quite interesting, first because it is a simple and robust mechanism based on the supplementary Lorentz force associated with the plasma current that compensates the cosmic-ray current, second because it leads to a turbulent field of large intensity; indeed this latter can become much larger than the ambient magnetic field. The theory indicates that the fraction of incoming energy flux converted into magnetic energy can reach $\xi_{B} \sim \beta_{s}$, which is a few percent in SNRs, where one defines

$$
\frac{B_{\mathrm{rms}}^{2}}{4 \pi}=\xi_{B} \rho_{u} V_{s}^{2} .
$$

A very important remark is that the efficiency of the Fermi process depends on the efficiency of the scattering process. By the way, the mechanism of Fermi acceleration is a simple 
Table 2 Comparison non-relativistic shocks and relativistic shocks

\begin{tabular}{ll}
\hline At non-relativistic shocks & At relativistic shocks \\
\hline Weak escape probability & Significant escape probability \\
Many cycles of weak energy gain & Few cycles of large energy gain \\
Power law distribution $\varepsilon^{-s}$ with $s \simeq 2$ & Power law distribution $\varepsilon^{-s}$ with $s \sim 2.3$ \\
Upstream distribution weakly anisotropic & Upstream distribution strongly anisotropic \\
Partial reflection at shock front & Partial reflection at shock front \\
Generation of MHD turbulence upstream & Generation of e.m. micro-turbulence upstream \\
Acceleration time $t_{a c c} \sim \tau_{s} / \beta_{s}^{2}$ & Acceleration time $t_{a c c} \sim \tau_{s}$ \\
\hline
\end{tabular}

process, but the scattering, that controls the efficiency of the acceleration process, is the main issue.

As for relativistic shocks, there are similarities and some differences with the nonrelativistic ones, as summarized in Table 2. There are strong arguments that there is a significant generation of magnetic turbulence at the external shock of a GRB ( $\mathrm{Li}$ and Waxman 2006) and there is an obvious power-law distribution of ultra-relativistic electrons that synchrotron radiate, with an index compatible with the theory of the Fermi process at ultrarelativistic shocks $(s=2.2-2.3)$. The ambient magnetic field is very low and at first approximation can be neglected. A remarkable work was published by Spitkovsky (2008) that fully validates the paradigm, combining three fundamental processes: the formation of a collisionless relativistic shock front with reflected particles, the generation of magnetic turbulence and the generation of a power-law distribution through the Fermi process. This is a PIC (Particles In Cell) simulation of the development of a collisionless shock in a pair plasma (electrons and positrons) that runs with a Lorentz factor $\Gamma_{s}$ of a few tens $\left(\Gamma_{s} \equiv\left(1-\beta_{s}^{2}\right)^{-1 / 2}\right)$. The flow of reflected particles interacts with the flow of passing particles leading to streaming-type instabilities, and the Weibel branch of instability describes the formation of intense small-scale magnetic filaments. The relevant scale of the physics is the inertial length (or skin depth) $\delta \equiv \frac{c}{\omega_{p}}$. The spatial growth of the magnetic microturbulence produces a partial reflection of the incoming particles, which allows the formation of a shock front, and self-consistently, the reflected particles generate the required level of micro-turbulence. Similarly as the non-relativistic case, conversion parameters $\xi_{\mathrm{cr}}, \xi_{B}$ can be defined in the ultra-relativistic case:

$$
\begin{aligned}
P_{\mathrm{cr}} & =\xi_{\mathrm{cr}} \rho_{u} \Gamma_{s}^{2} c^{2}, \\
\frac{B_{\mathrm{rms}}^{2}}{4 \pi} & =\xi_{B} \rho_{u} \Gamma_{s}^{2} c^{2} .
\end{aligned}
$$

And the simulations indicate that $\xi_{\mathrm{cr}} \sim 0.1$ and $\xi_{B} \sim 1-10 \%$, similarly to the non-relativistic case. The supra-thermal spectrum obtained in the simulation is close to the theoretical prediction with an index $s \simeq 2.4$. Similar results were obtained later with PIC simulation involving a plasma of electrons and ions of $(10 \ldots 100) m_{e}$ (Sironi and Spitkovsky 2009).

\subsection{Opening Phase Space with Finite Magnetization}

Many astrophysical shocks form in a plasma having a significant magnetization. The physics becomes more complex with a finite ambient mean field; it is controlled by the important 
“magnetization" parameter $\sigma$ :

$$
\sigma \equiv \frac{B_{t, f}^{2}}{4 \pi \rho_{u} \Gamma_{s}^{2} c^{2}}=\frac{B_{0}^{2} \sin ^{2} \theta_{B}}{4 \pi \rho_{u} c^{2}},
$$

where $B_{0}$ is the field measured in the upstream flow frame (generally the ambient field), and $B_{t, f}$ is the transverse component of the mean field measured in the shock frame. Like in non-relativistic shocks, the angle of the field lines with respect to the shock normal is very important. But whereas most non-relativistic shocks are in the so-called "sub-luminal" configuration, i.e. that the angle $\theta_{B}$ is not too close to $90^{\circ}$ and thus particles can flow along the field lines, in ultra-relativistic shocks, it suffices that the field angle $\theta_{B}$ be larger than $1 / \Gamma_{s}$ to prevent the return of particles to the upstream region. A generic ultra-relativistic shock is thus "supra-luminal", and the magnetic field in the front frame can be considered as almost perpendicular, because its transverse component is amplified by a factor $\Gamma_{s}$. This field orientation is a serious hindrance for the development of Fermi cycles. Neglecting for the time being any scattering process in a putative turbulence superimposed on the background field, the particle kinematics can be described as follows. A particle that enters the downstream flow of speed $c / 3$ is dragged by the frozen in magnetic field and cannot easily come back upstream; it can be shown that it can come back just one time (Lemoine et al. 2006). Once upstream, it eventually comes back downstream, but in a subset of phase space that does not allow it to make a second cycle. Now, it might be thought that a strong turbulence could provide efficient scattering allowing it to make several cycles. However, the typical interstellar turbulent field with a large-scale coherence length behaves like an ordered magnetic field for such particles, because their penetration length upstream $\left(\ell_{p}=m_{p} c^{2} / \Gamma_{s} e B_{0}\right.$, measured in the co-moving upstream frame) is much shorter than the coherence length of turbulence (Lemoine et al. 2006). In self-generated small scale turbulence, scattering might be efficient enough to trigger Fermi acceleration, see below.

The coherence length $\ell_{c}$ is formally defined as the range of the field correlation using the self-correlation function, $C(r)$. For an isotropic turbulent state we can write (it can easily be properly modified in the case of anisotropic turbulence):

$$
\ell_{c} \equiv \int_{0}^{\infty} C(r) \mathrm{d} r
$$

which can be expressed as an integral over the turbulence spectrum, and one finds that for a spectrum proportional to $k^{-\beta}$, the correlation length corresponds to large wavelengths for $1<\beta<2$, as is the case of a Kolmogorov spectrum; for $0 \leq \beta \leq 1$, the coherence length is in the shortest-wavelengths part of the spectrum.

Moreover, the expected duration of the cycle would be much shorter than the eddy turnover time of large-scale vortices. The requirements for efficient scattering off magnetic turbulence are quite challenging (Pelletier et al. 2009), for not only the intensity of the turbulent field must be much larger than the mean field, but also the coherence length must be shorter than a Larmor radius. When a scattering process develops, phase space is opened for operating a Fermi process if the scattering frequency is larger than the Larmor pulsation in the mean field. Short-scale turbulence leads to a scattering frequency $v_{s} \propto \varepsilon^{2}$, whereas the Larmor pulsation $\omega_{L} \propto \varepsilon$; thus the range of particle energies for which the phase space is unlocked and Fermi process operative, is such that $\varepsilon<\varepsilon_{\text {scatt }} \equiv Z e\left(\bar{B}^{2} / B_{0}\right) \ell_{c}$.

At high magnetization (say $\sigma>0.03$ ) the shock is formed by generation of an intense coherent wave through a Synchrotron Maser Instability due to a resonance with the loop of reflected particles (Hoshino and Arons 1991; Gallant et al. 1992). The electromagnetic 
wave propagating downstream is damped by synchrotron resonance and produces a thermal distribution. The wave propagating upstream carries away a fraction $\sim 0.1 \sigma$ of the incoming energy in the case of an $e^{+}-e^{-}$-plasma; in a $p^{+}-e^{-}$-plasma, an electrostatic wake field is generated that heats the electrons up to equipartition while slowing down protons (Hoshino and Arons 1991; Sironi and Spitkovsky 2011). The formation of a power-law distribution (Hoshino 2008) has not been confirmed as far as we know. The final word has not been given on these issues of course, because the simulations have been conducted so far in 1D (Hoshino and Arons 1991; Gallant et al. 1992) or 2D (Sironi and Spitkovsky 2011) over a limited amount of time. What happens in a more realistic 3D simulation, the dimensionality of which should allow more efficient cross-field transport, or on longer timescales, remain to be seen (see Jones et al. 1998).

According to Sironi and Spitkovsky (2011), at lower magnetization, nothing happens except the thermalization of protons $\left(T_{p} \simeq 0.2 \Gamma_{s} m_{p} c^{2}\right)$, until the magnetization reaches a very low critical value at which the Fermi process starts. As the magnetization decreases, indeed the precursor length scale increases, to the point where plasma microinstabilities triggered by the suprathermal particle population self-generate a small scale turbulence that can sustain the Fermi process (Lemoine \& Pelletier 2010). Actually, one needs a very low magnetic field to obtain an upstream penetration length of supra-thermal particles large enough for having a significant interaction of those particles with the incoming plasma and having a growth of micro-instabilities. The Fermi process works with the magnetic component of micro-turbulence at the inertial scale $\sim \delta \equiv c / \omega_{p i}$. In principle it starts at even smaller scale, the inertial scale of electrons, however electrons are efficiently heated by the electric component of micro-turbulence and then the precursor becomes composed of electrons and protons of similar relativistic mass, like a pair plasma. This is a very interesting outcome that simplifies the physics which rapidly evolves towards conditions similar to those occurring in a pair plasma. Thus the PIC simulations of pair plasma are also valuable to understand the physics of shocks in electron-proton plasmas. Then a distribution function displaying a thermal part and a supra-thermal part with a power law is obtained.

The transition towards the Fermi process is determined by the micro-instabilities that can grow when the upstream penetration of reflected particles is long enough. The fastest instabilities (Buneman instability, Oblique Two-Stream instability, see Bret et al. 2004) seem to essentially pre-heat the incoming electrons almost up to equipartition with protons. However, more simulations are necessary to clarify this important point. The generation of magnetic micro-turbulence by the Weibel instability, which is also studied in laboratory experiments, is thought to be the main ingredient to form collisionless shocks and to produce the Fermi process; however this is also under study by PIC simulations. The generation of magnetic micro-turbulence occurs when the magnetization parameter falls below the following critical value (Lemoine and Pelletier 2011a), as confirmed by numerical simulations (Sironi and Spitkovsky 2011):

$$
\sigma<\sigma_{\mathrm{crit}} \equiv \frac{\xi_{\mathrm{cr}}}{\Gamma_{s}^{2}}
$$

Numerical simulations show that the level reached by that Weibel turbulence is such that $\xi_{B}=1-10 \%$, which insures shock formation and Fermi process. Then there exists a large energy range for particle scattering when $\sigma \ll \xi_{B}^{2}$.

\subsection{The Micro-physics Aspect of GRB Termination Shocks}

The main issue with Fermi processes based on the scattering off micro-turbulence is that the scattering frequency decreases as $E^{-2}$. The performance of Fermi processes at non- 
relativistic shocks is determined by the scattering off large-scale, say Kolmogorov, turbulence which is fairly slow (much slower than the Larmor pulsation in the mean field) but decreases only as $E^{-1 / 3}$. Thus, if we compare the Fermi process at relativistic shocks with the process at non-relativistic shock, this is like the hare and the tortoise: the scattering, and thus the acceleration rhythm, at relativistic shocks is very fast at low energy and decreases rapidly as energy increases, whereas, at non-relativistic shocks, it is slow at low energy but continues at higher energies with a moderate decline of its efficiency.

\subsubsection{Electron Acceleration and Radiation}

The external shock that drives the afterglow emission of GRBs may give rise to an efficient acceleration of electrons if the external medium is weakly magnetized, for the reasons discussed previously. If electrons thermalize with protons (as reasonably expected), their temperature is already very high at the beginning of the afterglow: $T_{e} \sim T_{p} \simeq 0.2 \Gamma_{s} m_{p} c^{2}$, which corresponds to a few tens of $\mathrm{GeV}$. Intense short-scale magnetic turbulence develops because the interstellar magnetization parameter is very low, $\sigma \sim 10^{-9}$, whereas the critical value $\sigma_{\text {crit }} \sim 10^{-6}$, with $\Gamma_{s} \sim 300$.

What kind of radiation can be expected in such small-scale field, much more intense than the mean field? This depends on a so-called "wiggler" parameter $a$ :

$$
a \equiv \frac{e B_{\mathrm{rms}} \ell_{c}}{m_{e} c^{2}} \sim \xi_{B}^{1 / 2} \Gamma_{s} \frac{m_{p}}{m_{e}} .
$$

This parameter measures the capability of the magnetic force to deflect a relativistic electron of Lorentz factor $\gamma$ by an angle $1 / \gamma$ (which is the reason why $\gamma$ does not appear in the definition). If $a>1$, then the magnetic field produces a single deflection of the electron in the emission cone of half angle $1 / \gamma$, whereas if $a<1$ the electron can undergo several wiggles in the emission cone. When $a$ is large, the emission behaves like normal synchrotron radiation in a mean field, except that there is no polarization. When $a$ is small, the emission is of "jitter" type (Medvedev 2000). Thus the emission caused by shocked and accelerated electrons at a relativistic shock is "synchrotron-like", and the analysis of the emitted spectrum provides a diagnostic of the magnetic turbulence.

It is quite remarkable that there exists an almost universal energy limit for the electron radiating in the intense small scale field (in agreement with Kirk and Reville 2010):

$$
\gamma_{\max } \approx\left(\frac{4 \pi e^{2} \ell_{c}}{\sigma_{T} m_{e} c^{2}}\right)^{1 / 3} \simeq\left(\frac{m_{p}}{n m_{e} r_{e}^{3}}\right)^{1 / 6} \approx 10^{6} .
$$

The corresponding maximum energy for the photons emitted in the quasi-homogeneous field is

$$
E_{\gamma, \max } \sim \sqrt{\pi} \xi_{B}^{1 / 2} \frac{\Gamma_{s}^{2}}{\gamma_{\max }} \frac{m_{p} c^{2}}{\alpha_{f}} \sim 2 \times\left(\frac{\xi_{B}}{10^{-2}}\right)^{1 / 2}\left(\frac{\Gamma_{s}}{300}\right)^{2} \mathrm{GeV},
$$

where $\alpha_{f}$ is the fine structure constant. The account for magnetic fluctuations of scales larger than the synchrotron emission formation length results in the photon spectra extended beyond the limit given by Eq. (22) (see Bykov et al. 2012). Thus a single synchrotron-like spectrum extending up to several $\mathrm{GeV}$, even possibly a few tens, can be expected and is in fact compatible with observations. So the performance of relativistic shocks for electron acceleration and radiation appears very satisfactory. The conversion factor into radiation is $\xi_{\text {rad }} \sim \xi_{B} \sigma_{T} n_{0} r_{s}\left\langle\gamma_{e}^{2}\right\rangle$, and at the beginning of the afterglow $\xi_{\text {rad }} \sim \xi_{B} \sim 1-10 \%$. 


\subsubsection{Proton Acceleration Limited by the Fast Decay of Scattering}

Protons are expected to be accelerated at least as efficiently as electrons at ultra-relativistic shock waves. However, as mentioned previously, the ultra-relativistic Fermi process appears unable to push protons up to energies in excess of $\sim 10^{17}-10^{18} \mathrm{eV}$, because the scattering time and thus the acceleration time increase with $E^{2}$ in the self-generated turbulent field, or scale with $E$, but then in the background unamplified field.

Using one or the other, one does not find numbers significantly different from the limit associated to the mean field discussed in Sect. 5.2 (Eq. (9)): $E_{\max }=Z \Gamma_{S} e B_{0} R_{S} \simeq Z \times(0.3$. $\left.10^{7} \mathrm{GeV}\right)$. Thus, although an energy of order $10^{16} \mathrm{eV}$ is achieved, which is something, the result is far from reaching the UHE-range.

Precise performances of mildly or sub-relativistic shocks are not yet known and require more numerical simulations. However, some reasonable estimates are permitted by extrapolating what we know about the two extremes: non-relativistic and ultra-relativistic shocks. The main guess is that we can expect a magnetic-field amplification at shocks with a conversion factor $\xi_{B}=1-10 \%$, occurring in MHD regime without severe limitation due to the super-luminal configuration, especially for oblique internal shocks (termination shocks in the hot spots of FR2 jets might be super-luminal). These assumptions can be applied to internal shocks of AGN jets (in particular in Blazars jets), and to internal shocks of GRBs, as already discussed in Sect. 5.3.

\subsection{Conclusion and Prospect}

The triangular dependence of collisionless shock structure with a reflecting barrier for a part of incoming particles, with generation of supra-thermal particles and the generation of magnetic turbulence is a successful paradigm that applies to astrophysical shocks, both nonrelativistic and relativistic. Numerical and theoretical works are making significant progress for both understanding the physics and providing quantitative results useful for astrophysical investigations. This includes not only the spectral index and cut off of the distribution of accelerated particles, but also the efficiency factors for the conversion into cosmic rays, magnetic turbulence and radiation. We have seen only the beginning of this line of study, which requires more PIC simulations and new types of hybrid codes involving relativistic MHD coupled with PIC codes for cosmic rays.

The new results that have already been obtained are important. First, the strong amplification of the magnetic field at SNRs received theoretical and numerical support; the astrophysical consequences are interesting, especially for our understanding of the Galactic contribution of the cosmic-ray spectrum. Secondly, current state-of-the-art PIC simulations indicate that the Fermi process does not operate at ultra-relativistic shocks with magnetization of order unity, which is supposed to be a frequent situation in high-energy astrophysics, as for instance in FR2 hot spots, in blazars, in pulsar wind nebulae. Such simulations need however to be extended both to higher dimensionality and to larger space-time domains before a definite conclusion can be reached. In particular, the issue of the non stationarity and/or corrugation of the shock front in relativistic regime should be investigated. Also the role of magnetic reconnections in the shock vicinity is a very important new topic whose investigation is just starting. Whether and how acceleration proceeds in the mildly-relativistic regime also remains open for study. Thirdly the radiation processes that operate in most high-energy astrophysical sources involve relativistic electrons scattered in an intense shortscale magnetic turbulence; this leads to a renewed interest in the radiation physics with a view to use it as a diagnostic of the magnetic turbulent state. 
The new trend in these topics is the important role imputed to micro-physics phenomena, which have a direct astrophysical impact. These developments incite interest in several other communities, including space-plasma physics, laser-plasma physics, astroparticle, and highenergy astrophysics. We live in exciting times.

Acknowledgements A.M.B. was supported in part by the Russian government grant 11.G34.31.0001 to Sankt-Petersburg State Politechnical University, and also by the RAS and RAS Presidium Programs and by the RFBR grant 11-02-12082. N.G. acknowledges scientific discussions and assistance by J. Cannizzo. M.P. is supported by the 'Helmholtz Alliance for Astroparticle Phyics HAP' funded by the Initiative and Networking Fund of the Helmholtz Association. M.L. and G.P. acknowledge financial support of the PEPS/PTI program of the CNRS-INP.

\section{References}

R.U. Abbasi, T. Abu-Zayyad, M. Al-Seady et al., Phys. Rev. Lett. 104, 161101 (2010)

A.A. Abdo, M. Ackermann, M. Arimoto et al., Science 323, 1688 (2009a)

A.A. Abdo, M. Ackermann, M. Ajello et al., Nature 462, 331 (2009b)

A.A. Abdo et al., Phys. Rev. Lett. 104, 101101 (2010)

J. Abraham, M. Aglietta, I.C. Aguirre et al., Nucl. Instrum. Methods Phys. Res. A 523, 50 (2004)

J. Abraham, P. Abreu et al., Astropart. Phys. 29, 188 (2008)

J. Abraham, P. Abreu, M. Aglietta et al., Phys. Rev. Lett. 104, 091101 (2010b)

J. Abraham, P. Abreu, M. Aglietta et al., Phys. Lett. B 685, 239 (2010a)

P. Abreu, M. Aglietta et al., Astropart. Phys. 34, 314 (2010)

P. Abreu, M. Aglietta et al., Astropart. Phys. 34, 627 (2011)

V.A. Acciari, E. Aliu, T. Arlen et al., Science 325, 444 (2009)

V.A. Acciari, E. Aliu, T. Arlen et al., Astrophys. J. 738, 25 (2011)

A. Achterberg, Astron. Astrophys. 119, 274 (1983)

A. Achterberg, Y.A. Gallant, J.G. Kirk, A.W. Guthmann, Mon. Not. R. Astron. Soc. 328, 393 (2001)

F.A. Aharonian, A.G. Akhperjanian, J.A. Barrio et al., Astron. Astrophys. 349, 11 (1999)

R. Aloisio, V. Berezinsky, P. Blasi et al., Astropart. Phys. 27, 76 (2007)

R. Aloisio, V. Berezinsky, A. Gazizov, Astropart. Phys. 34, 620 (2011)

W.D. Apel, J.C. Arteaga, A.F. Badea et al., Astropart. Phys. 31, 86 (2009)

J. Arons, Astrophys. J. 589, 871 (2003)

S.D. Barthelmy, G. Chincarini, D.N. Burrows et al., Nature 438, 994 (2005)

J. Bednarz, M. Ostrowski, Phys. Rev. Lett. 80, 3911 (1998)

A.R. Bell, Mon. Not. R. Astron. Soc. 353, 550 (2004)

A.R. Bell, Mon. Not. R. Astron. Soc. 358, 181 (2005)

A.R. Bell, S.G. Lucek, Mon. Not. R. Astron. Soc. 321, 433 (2001)

E.G. Berezhko, Astrophys. J. Lett. 684, L69 (2008)

V. Berezinsky, A. Gazizov, S. Grigorieva, Phys. Rev. D 74, 043005 (2006)

E. Berger, Astrophys. J. 722, 1946 (2010)

E. Berger, P.A. Price, S.B. Cenko et al., Nature 438, 988 (2005)

R.D. Blandford, R.L. Znajek, Mon. Not. R. Astron. Soc. 179, 433 (1977)

P.L. Biermann, P.A. Strittmatter, Astrophys. J. 322, 643 (1987)

R.D. Blandford, C.F. McKee, Phys. Fluids 19, 1130 (1976)

J.S. Bloom, J.X. Prochaska, D. Pooley et al., Astrophys. J. 638, 354 (2006)

A. Bret, M.-C. Firpo, C. Deutsch, Phys. Rev. E 70, 046401 (2004)

D.N. Burrows, D. Grupe, M. Capalbi et al., Astrophys. J. 653, 468 (2006)

A.M. Bykov, Space Sci. Rev. 99, 317 (2001)

A.M. Bykov, P. Meszaros, Astrophys. J. Lett. 461, L37 (1996)

A.M. Bykov, I.N. Toptygin, Phys. Usp. 36, 1020 (1993)

A.M. Bykov, R.A. Treumann, Astron. Astrophys. Rev. 19, 42 (2011)

A.M. Bykov, S.M. Osipov, D.C. Ellison, Mon. Not. R. Astron. Soc. 410, 39 (2011)

A.M. Bykov, G.G. Pavlov, A.V. Artemyev, Y.A. Uvarov, Mon. Not. R. Astron. Soc. Lett. 421, L67 (2012)

G. Cassam-Chenaï, A. Decourchelle, J. Ballet et al., Astron. Astrophys. 414, 545 (2004)

A. Celotti, G. Ghisellini, M. Chiaberge, Mon. Not. R. Astron. Soc. 321, L1 (2001)

E. Costa, F. Frontera, J. Heise et al., Nature 387, 783 (1997)

F. Daigne, R. Mochkovitch, Mon. Not. R. Astron. Soc. 296, 275 (1998) 
D. Eichler, M. Pohl, Astrophys. J. Lett. 738, L21 (2011)

D. Eichler, D. Guetta, M. Pohl, Astrophys. J. 722, 543 (2010)

D.C. Ellison, G.P. Double, Astropart. Phys. 18, 213 (2002)

G. Ferrand, A. Marcowith, Astron. Astrophys. 510, A101 (2010)

W. Fong, E. Berger, D.B. Fox, Astrophys. J. 708, 9 (2010)

D.B. Fox, D.A. Frail, P.A. Price et al., Nature 437, 845 (2005)

D.A. Frail, S.R. Kulkarni, L. Nicastro, M. Feroci, G.B. Taylor, Nature 389, 261 (1997)

D.A. Frail, S.R. Kulkarni, R. Sari et al., Astrophys. J. Lett. 562, L55 (2001)

Y.A. Gallant, A. Achterberg, Mon. Not. R. Astron. Soc. 305, L6 (1999)

Y.A. Gallant, M. Hoshino, A.B. Langdon, J. Arons, C.E. Max, Astrophys. J. 391, 73 (1992)

A.B. Garson III, M.G. Baring, H. Krawczynski, Astrophys. J. 722, 358 (2010)

N. Gehrels, G. Chincarini, P. Giommi et al., Astrophys. J. 611, 1005 (2004)

N. Gehrels, C.L. Sarazin, P.T. O’Brien et al., Nature 437, 851 (2005)

N. Gehrels, E. Ramirez-Ruiz, D.B. Fox, Annu. Rev. Astron. Astrophys. 47, 567 (2009)

D. Giannios, H.C. Spruit, Astron. Astrophys. 430, 1 (2005)

D. Giannios, D.A. Uzdensky, M.C. Begelman, Mon. Not. R. Astron. Soc. 402, 1649 (2010)

D. Götz, P. Laurent, F. Lebrun, F. Daigne et al., Astrophys. J. Lett. 695, L208 (2009)

D. Grupe, D.N. Burrows, S.K. Patel et al., Astrophys. J. 653, 462 (2006)

D.E. Harris, H. Krawczynski, Astrophys. J. 565, 244 (2002)

D.E. Harris, H. Krawczynski, Annu. Rev. Astron. Astrophys. 44, 463 (2006)

M. Hillas, Annu. Rev. Astron. Astrophys. 22, 425 (1984)

J. Hjorth, D. Watson, J.P.U. Fynbo et al., Nature 437, 859 (2005)

M. Hoshino, Astrophys. J. 672, 940 (2008)

M. Hoshino, J. Arons, Phys. Fluids B 3, 818 (1991)

F.C. Jones, J.R. Jokipii, M.G. Baring, Astrophys. J. 509, 238 (1998)

M. Kachelrieß, S. Ostapchenko, R. Tom's, Publ. Astron. Soc. Aust. 27, 482 (2010)

M. Kachelriess, D.V. Semikoz, Phys. Lett. B 634, 143 (2006)

Y. Kaneko, E. Ramirez-Ruiz, J. Granot et al., Astrophys. J. 654, 385 (2007)

Y. Kaneko, M.M. González, R.D. Preece, B.L. Dingus, M.S. Briggs, Astrophys. J. 677, 1168 (2008)

H. Kang, J.P. Rachen, P.L. Biermann, Mon. Not. R. Astron. Soc. 286, 257 (1997)

H. Kawai, S. Yoshida, H. Yoshii et al., Nucl. Phys. B, Proc. Suppl. 175, 221 (2008)

J.G. Kirk, B. Reville, Astrophys. J. Lett. 710, L16 (2010)

J.G. Kirk, F.M. Rieger, A. Mastichiadis, Astron. Astrophys. 333, 452 (1998)

J.G. Kirk, A.W. Guthmann, Y.A. Gallant, A. Achterberg, Astrophys. J. 542, 235 (2000)

J.G. Kirk, Y. Lyubarsky, J. Petri, in Astrophysics and Space Science Library, vol. 357, ed. by W. Becker (2009), p. 421

R.W. Klebesadel, I.B. Strong, R.A. Olson, Astrophys. J. Lett. 182, L85 (1973)

K. Kotera, A.V. Olinto, Annu. Rev. Astron. Astrophys. 49, 119 (2011)

C. Kouveliotou, C.A. Meegan, G.J. Fishman et al., Astrophys. J. Lett. 413, L101 (1993)

H. Krawczynski, R. Sambruna, A. Kohnle et al., Astrophys. J. 559, 187 (2001)

H. Krawczynski, P.S. Coppi, F. Aharonian, Mon. Not. R. Astron. Soc. 336, 721 (2002)

L. Kuiper, W. Hermsen, G. Cusumano et al., Astron. Astrophys. 378, 918 (2001)

P. Kumar, Astrophys. J. Lett. 523, L113 (1999)

P. Kumar, R. Barniol Duran, Mon. Not. R. Astron. Soc. 409, 226 (2010)

P.O. Lagage, C. Cesarsky, Astron. Astrophys. 125, 249 (1983a)

P.O. Lagage, C. Cesarsky, Astron. Astrophys. 118, 223 (1983b)

T. Le, C.D. Dermer, Astrophys. J. 661, 394 (2007)

M. Lemoine, G. Pelletier, Astrophys. J. Lett. 589, L73 (2003)

M. Lemoine, G. Pelletier, Mon. Not. R. Astron. Soc. 402, 321 (2010)

M. Lemoine, G. Pelletier, Mon. Not. R. Astron. Soc. 417, 1148 (2011a)

M. Lemoine, G. Pelletier (2011b). arXiv:1111.7110

M. Lemoine, E. Waxman, J. Comp. Astropart. Phys. 11, 9 (2009)

M. Lemoine, G. Pelletier, B. Revenu, Astrophys. J. Lett. 645, L129 (2006)

E.M. Levesque, Am. Inst. Phys. Conf. Ser. 1358, 271 (2011)

E.M. Levesque, L.J. Kewley, E. Berger, H. Jabran Zahid, Astron. J. 140, 1557 (2010)

Z. Li, E. Waxman, Astrophys. J. 651, 328 (2006)

S.G. Lucek, A.R. Bell, Mon. Not. R. Astron. Soc. 314, 65 (2000)

M. Lyutikov, R. Blandford, in Astronomical Society of the Pacific Conference Series, vol. 312, ed. by M. Feroci, F. Frontera, N. Masetti, L. Piro (2004), p. 449

A.I. MacFadyen, S.E. Woosley, Astrophys. J. 524, 262 (1999)

M.A. Malkov, P.H. Diamond, Phys. Plasmas 8, 5 (2001) 2401 
M.L. McConnell, A.A. Zdziarski, K. Bennett et al., Astrophys. J. 572, 984 (2002)

J.F. McKenzie, H.J. Voelk, Astron. Astrophys. 116, 191 (1982)

J.C. McKinney, Astrophys. J. Lett. 630, L5 (2005)

J.C. McKinney, R. Narayan, Mon. Not. R. Astron. Soc. 375, 513 (2007a)

J.C. McKinney, R. Narayan, Mon. Not. R. Astron. Soc. 375, 531 (2007b)

J.C. McKinney, D.A. Uzdensky, Mon. Not. R. Astron. Soc. 419, 573 (2012)

M.V. Medvedev, Astrophys. J. 540, 704 (2000)

M.V. Medvedev, A. Loeb, Astrophys. J. 526, 697 (1999)

P. Mészáros, Rep. Prog. Phys. 69, 2259 (2006)

P. Mészáros, M.J. Rees, Astrophys. J. Lett. 482, L29 (1997)

P. Mimica, Mon. Not. R. Astron. Soc. 2427 (2012)

Y. Mizuno, M. Pohl, J. Niemiec et al., Astrophys. J. 726, 62 (2011)

K. Murase, K. Ioka, S. Nagataki, T. Nakamura, Astrophys. J. Lett. 651, L5 (2006)

K. Murase, S. Inoue, S. Nagataki, Astrophys. J. Lett. 689, L105 (2008)

K. Murase, P. Mészáros, B. Zhang, Phys. Rev. D 79, 103001 (2009)

K. Murase, K. Asano, T. Terasawa, P. Mészáros, Astrophys. J. 746, 164 (2012)

J. Niemiec, M. Ostrowski, M. Pohl, Astrophys. J. 650, 1020 (2006)

K.I. Nishikawa, J. Nimiec, M. Medvedev, B. Zhang et al., Int. J. Mod. Phys. D 19, 715-721 (2010)

C. Norman, D. Melrose, A. Achterberg, Astrophys. J. 454, 60 (1995)

A. Panaitescu, M. Spada, P. Mészáros, Astrophys. J. Lett. 522, L105 (1999)

A. Pe’Er, K. Murase, P. Mészáros, Phys. Rev. D 80, 123018 (2009)

G. Pelletier, M. Lemoine, A. Marcowith, Astron. Astrophys. 453, 181 (2006)

G. Pelletier, M. Lemoine, A. Marcowith, Mon. Not. R. Astron. Soc. 393, 587 (2009)

E.S. Perlman, J.A. Biretta, F. Zhou, W.B. Sparks, F.D. Macchetto, Astron. J. 117, 2185 (1999)

T. Piran, Rev. Mod. Phys. 76, 1143 (2004)

I. Plotnikov, G. Pelletier, M. Lemoine, Astron. Astrophys. 532, 68 (2011)

M. Pohl, D. Eichler, Astrophys. J. 742, 114 (2011)

M. Pohl, R. Schlickeiser, Astron. Astrophys. 354, 395 (2000)

M. Pohl, I. Lerche, R. Schlickeiser, Astron. Astrophys. 383, 309 (2002)

M. Pohl, H. Yan, A. Lazarian, Astrophys. J. Lett. 626, L101 (2005)

V.S. Ptuskin, S.I. Rogovaya, V.N. Zirakashvili (2011). arXiv:1105.4491

V. Ptuskin, V. Zirakashvili, E.-S. Seo, Astrophys. J. 718, 31 (2010)

J.P. Rachen, P.L. Biermann, Astron. Astrophys. 272, 161 (1993)

M.J. Rees, P. Meszaros, Astrophys. J. Lett. 430, L93-L96 (1994)

B. Reville, J.G. Kirk, P. Duffy, Plasma Phys. Control. Fusion 48, 1741 (2006)

R. Salvaterra, M. Della Valle, S. Campana et al., Nature 461, 1258 (2009)

R. Sari, T. Piran, R. Narayan, Astrophys. J. Lett. 497, L17 (1998)

L. Sironi, A. Spitkovsky, Astrophys. J. 698, 1523 (2009)

L. Sironi, A. Spitkovsky, Astrophys. J. 726, 75 (2011)

J. Skilling, Mon. Not. R. Astron. Soc. 173, 245 (1975)

A. Spitkovsky, Astrophys. J. Lett. 682, L5 (2008)

H.C. Spruit, F. Daigne, G. Drenkhahn, Astron. Astrophys. 369, 694-705 (2001)

F. Takahara, Prog. Theor. Phys. 83, 1071 (1990)

T. Takahashi et al., Astrophys. J. Lett. 542, 105 (2000)

N.R. Tanvir, D.B. Fox, A.J. Levan et al., Nature 461, 1254 (2009)

F. Tavecchio, L. Maraschi, R.M. Sambruna, C.M. Urry, Astrophys. J. Lett. 544, L23 (2000)

C. Thompson, Mon. Not. R. Astron. Soc. 270, 480 (1994)

M.S. Turner et al., Report to the National Academy of Science (2002)

V.V. Usov, Nature 357, 472-474 (1992)

J. van Paradijs, P.J. Groot, T. Galama et al., Nature 386, 686 (1997)

M. Vietri, Astrophys. J. 453, 883 (1995)

J.S. Villasenor, D.Q. Lamb, G.R. Ricker et al., Nature 437, 855 (2005)

A. Vladimirov, D.C. Ellison, A. Bykov, Astrophys. J. 652, 1246 (2006)

X.-Y. Wang, S. Razzaque, P. Mészáros, Z.-G. Dai, Phys. Rev. D 76, 083009 (2007)

E. Waxman, Phys. Rev. Lett. 75, 386 (1995)

E. Waxman, Astrophys. J. 606, 988 (2004)

E. Waxman, Phys. Scr. T 121, 147 (2005) 
E. Waxman (2010). arXiv:1010.5007

D.G. Wentzel, Annu. Rev. Astron. Astrophys. 12, 71 (1974)

M. Yamada, R. Kulsrud, H. Ji, Rev. Mod. Phys. 82, 603 (2010)

D. Yonetoku, T. Murakami, S. Gunji, T. Mihara et al., Astrophys. J. Lett. 743, L30 (2011)

B. Zhang, H. Yan, Astrophys. J. 726, 90 (2011)

W. Zhang, S.E. Woosley, A.I. MacFadyen, Astrophys. J. 586, 356 (2003) 